\title{
Application of the Modified Inverse Design Method in the Optimization of the Runner Blade of a Mixed-Flow Pump
}

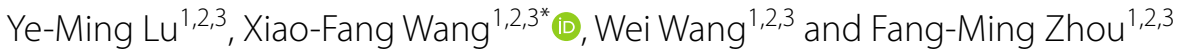

\begin{abstract}
To improve the design speed and reduce the design cost for the previous blade design method, a modified inverse design method is presented. In the new method, after a series of physical and mathematical simplifications, a sail-like constrained area is proposed, which can be used to configure different runner blade shapes. Then, the new method is applied to redesign and optimize the runner blade of the scale core component of the 1400-MW canned nuclear coolant pump in an established multi-optimization system compromising the Computational Fluid Dynamics (CFD) analysis, the Response Surface Methodology (RSM) and the Non-dominated Sorting Genetic Algorithm-II (NSGA-II). After the execution of the optimization procedure, three optimal samples were ultimately obtained. Then, through comparative analysis using the target runner blade, it was found that the maximum efficiency improvement reached $1.6 \%$, while the head improvement was about 10\%. Overall, a promising runner blade inverse design method which will benefit the hydraulic design of the mixed-flow pump has been proposed.
\end{abstract}

Keywords: Optimization, Mixed-flow pump, Inverse design method, Runner blade, Nuclear coolant

\section{Introduction}

Mixed-flow pumps have the characteristics of both centrifugal pumps and axial pumps. They are widely used in various areas, such as industrial chemistry, industrial water applications, nuclear industry. As the heart of the power equipment in these areas, mixed-flow pumps can provide stable energy for the circulation of fluid medium. The need for designing a higher efficiency pump with lower energy consumption is increasing with the current advocacy for green power, nowadays [1]. Accordingly, an effective design method for configuring the runner blade of the pump for high efficiency and reasonable high head would be valuable.

To design and optimize the blade shape, the distribution of the structural parameters, for instance the blade angles, were directly adjusted in previous studies [2-4]. Unfortunately, this approach is highly dependent on the

\footnotetext{
*Correspondence: dlwxf@dlut.edu.cn

${ }^{1}$ School of Energy and Power Engineering, Dalian University

of Technology, Dalian 116000, China

Full list of author information is available at the end of the article
}

designers' experiences. Additionally, a fair number of design parameters would need to be selected to be optimized in order to get a new runner blade that is quite different from the target. Instead of geometrically optimizing the blade with blade angles, an important inverse design method was proposed by Borges [5] to design a blade under incompressible conditions, which was subsequently extended to compressible conditions by Zangeneh $[6,7]$. In this inverse design approach, the blade shape was represented as sheets of blade loading with the approximate distribution controlled by specific variables at the hub and shroud sides, such a design method has already been adopted in the TURBODesign software. Using this sequential design method in the software, several optimal pumps have been successfully obtained $[8,9]$. However, some problems still remain waiting to be solved. First, the blade shape is simply controlled by two cross sections, namely, the hub section and the shroud section, and this so-called 2D blade profile can only configure a blade with straight leading edge instead of a complex bend leading edge. Nevertheless, the leading edge affects the cavitation and secondary flows [10], 
thus a design method which can present a complex leading edge would offer extra choices for improving the flow characteristics. Moreover, the ranges for the design variables are not clearly defined. Taking the design method in the TURBODesign software as an example, seven independent variables $[8,9]$, namely, NCs, NCh, NDs, NDh, SLOPEh, SLOPEs, LEh and LEs, are adopted, and without obvious definition for the range of these variables, a large number of samples would have to be generated before optimization, which would require a considerable amount of time and computing resource.

The shortcomings of the previous design methods, thus indicates that the more cross sections controlling the blade shape (for configuring the complex blade) and the fewer design variables (for quickly designing blade), the better the design method would be. However, the number of cross sections and the number of variables have an inverse proportional relationship. In some traditional design methods [11], even four or five cross sections are adopted, but they can just configure a few blade shapes due to the excessive number of variables located on the cross sections. To increase the control sections and decrease the number of variables, a modified design method based on the previous research is proposed here. The scholars associated with such research proposed the idea of using swirl velocity distribution to design the runner blade and have carried out relevant works [12, 13]. However, hardly any optimization work has adopted this method before. Besides, Bing [14] tried to specify the constrained variables to control the swirl velocity distribution, but the design variables have not been effectively limited. As for the modified design method in this paper, a sail-like constrained area controlling the design variables is deduced by some physical and mathematical simplifications based on Bing's work, using just three points located in the constrained area are used to configure the blade by controlling the cross sections. Ultimately, the modified inverse design method is effectively applied in a multi-optimization system to verify its effectiveness.

\section{Modified Inverse Design Method}

The Quasi-three-dimensional (Q3D) method which takes limited time and has successfully been applied to design the blade in previous studies $[9,12]$. As for the Q3D theory, instead of taking the iteration computation of the $S_{1}$ surface (blade-to-blade) and $S_{2}$ (hub-to-shroud) surface, it takes the representative mean $S_{2}$ surface $\left(S_{2 m}\right)$ to provide variables for the calculation of the $S_{1}$ surface in the iterative process. To get the flow variables on the $S_{2 m}$ surface, the preliminary calculation was performed by the given meridional shape, the blocking factor (determined by the blade numbers, blade thickness, etc.), operating conditions (including mass flow, temperature, pressure, etc.) according to the 2-D streamline curvature method $[15,16]$ or Computational Fluid Dynamics (CFD) [17]. Compared with the 2-D streamline curvature method, the CFD method is much more accurate [17], thus, it was adopted here to calculate the average-wised variables on $\mathrm{S}_{2 \mathrm{~m}}$ surface.

\subsection{Basic Runner Blade Design Theory}

To design the impeller of a mixed-flow pump, the following factors need to be considered: (I) meridional channel shape including a leading contour; (II) blade thickness; (III) blade number; (IV) staking condition; (V) loading pressure [9] or swirl velocity [12, 17]. As mentioned in the title, this study is mainly concerned with designing the blade, so that factor (I) and factor (III) remain the same as the original target impeller which will be introduced in Section 4.1. When compared with other factors, factor (II) has a faintness influence, so it was kept unchanged. As for factor (IV), the blade shape here can be controlled by three cross sections consisting of the hub side, the middle and the shroud side, which is shown in Figure 1(b). In Zangeneh's design theory, the loading pressure of factor $(\mathrm{V})$ is given as follows [6, 7]:

$$
p^{+}-p^{-}=(2 \pi / B) \rho V_{m} \frac{\partial\left(r V_{\theta}(s)\right)}{\partial s},
$$

where $p^{+}$and $p^{-}$represent static pressure on the pressure and suction side of the blade, $B$ is the blade number, $\rho$ is the density and $V_{m}$ is the pitch-wise averaged meridional velocity on the $\mathrm{S}_{2 \mathrm{~m}}$ surface.

Regarding Zangenh's theory, after settling down the meridian shape, blade thickness and blade number, the preliminary averaged meridional velocity can be calculated at first. And with the preliminary meridional velocity and a given distribution of swirl velocity, the loading pressure can then be calculated to configure the blade shape according to Eq. (1). Afterwards, with the newly configured blade, the meridional velocity can be recalculated. Additionally, the configured blade can be subjected to minor modifications according to the new meridional velocity and the established swirl velocity before. With the iterative modification above, the blade shape can be finally established until the variables remain stable.

As a result, the averaged meridional velocity is the intermediate variable and becomes gradually stable during the design process above, so that the swirl velocity becomes the unique variable determining the loading pressure as well as the blade shape. Regarding the factor (V), the swirl velocity can also take the place of the loading pressure distribution in determining the runner blade shape [17]. 


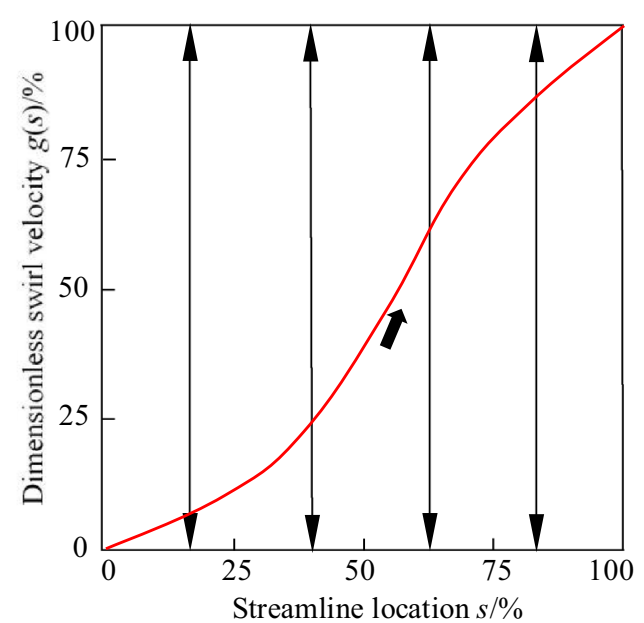

a Sketch presentation of the swirl velocity distribution

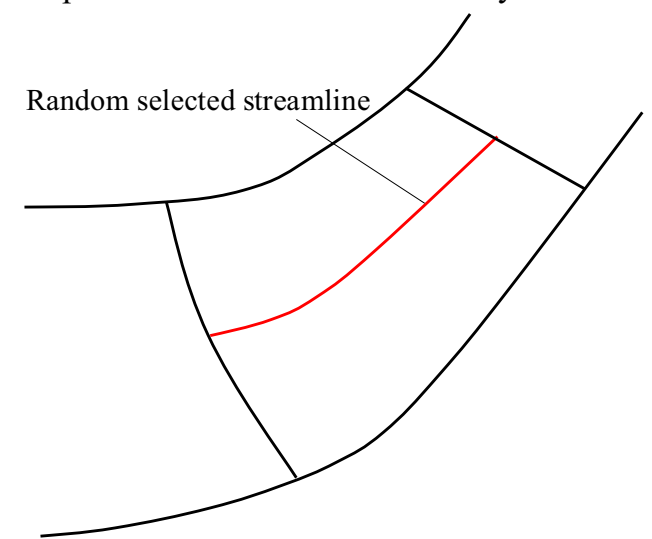

b Random selected streamline

Figure 1 Physical distribution law for the swirl velocity

Unlike Zangeneh's design theory, which uses the loading pressure to design the blade shape, the new design method uses swirl velocity to directly determine the geometric variables. Here, the distribution of the swirl velocity is established first, and then it is combined with another traditional equation instead of using Eq. (1). Such equation can calculate the wrap angles directly, and is given as $[6,12]$ :

$$
\theta=\int \frac{\omega r^{2}-r V_{\theta}(s)}{V_{m} r^{2}} \mathrm{~d} s,
$$

where $\omega$ represents the rotation velocity of the pump, and $r$ is the radial coordinate.

After the calculation of the wrap angles, the results are then imported into a three-dimensional modelling software among with the other established variables, such as blade thickness, meridional shape and blade number. Accordingly, it can be concluded that the distribution of swirl velocity is the key factor to design the runner blade shape, and its distribution law will be discussed next.

\subsection{Derivation of the Constrained Area}

As mentioned above, swirl velocity plays an extremely important role in determining the blade shape on the condition that the meridional shape, blade thickness and blade number are settled first. The distribution for swirl velocity along streamline can be described as follows [14]:

$$
r V_{\theta}(s)=r_{1} V_{\theta 1}+g(s)\left(r_{2} V_{\theta 2}-r_{1} V_{\theta 1}\right),(0 \leq s \leq 1),
$$

where $r_{1} V_{\theta 1}$ and $r_{2} V_{\theta 2}$ denote the swirl velocity at the inlet and outlet respectively, $g(s)$ is the non-dimensional swirl velocity. According to pre-design variables from the target and the one-dimensional theory, $r_{1} V_{\theta 1}$ and $r_{2} V_{\theta 2}$ can be determined by the following equations $[18,19]$ :

$$
\begin{aligned}
& r_{1} V_{\theta 1}=r_{1}^{2} \omega-\frac{V_{m 1} r_{1}}{\tan \beta_{1}}, \\
& r_{2} V_{\theta 2}=\frac{(1+\gamma) g H_{d}}{\omega \eta_{h}}+r_{1} V_{\theta 1}, \\
& \beta_{1} \approx \arctan \frac{V_{1 m}}{u_{1}},
\end{aligned}
$$

where $\beta_{1}$ is the inlet blade angle and can be calculated by the established variables from the target pump; $H_{\mathrm{d}}$ is the design target head; $V_{\mathrm{m} 1}$ is the meridional velocity at the inlet; $\gamma$ is the correction factor; $\eta_{h}$ is the hydraulic efficiency; $u_{1}$ is the circumferential velocity at the inlet.

Then, how to settle down the distribution of $g(s)$ would be a crucial step for the calculation of the swirl velocity. Actually, $g(s)$ can also be expressed by the quartic polynomial [17]:

$$
g(s)=a s^{4}+b s^{3}+c s^{2}+d s+e,
$$

where $a, b, c, d, e$ represent the coefficients.

Figure 1(a) gives the physical distribution law for the dimensionless swirl velocity $[17,20]$, which ranges from 0 to 1 and has an increasing trend along streamline. The distribution law can be expressed as the following mathematical constraints:

$$
\begin{aligned}
& g(0)=0, \\
& g(1)=1, \\
& \partial(g(0)) / \partial s=0 \text { (Kutta-Joukowski condition), }
\end{aligned}
$$




$$
\begin{aligned}
& \partial(g(1)) / \partial s=P,(P \text { is constant, } P \geq 0) \\
& \partial(g(s)) /\left.\partial s\right|_{s \in[0,1]} \geq 0 \\
& \left.g(s)\right|_{s \in[0,1]} \leq 1 .
\end{aligned}
$$

where Eq. (10) is from Ref. [6]; Eq. (11) is from Ref. [17]; Eq. (12) is from Ref. [14].

With the combination of Eqs. (7)-(13), they can be further simplified as:

$$
\left\{\begin{array}{l}
b=-2 a+P-2, c=a-P+3, \\
d=0, e=0, \\
(s-1)(2 s-1) a \geq(3-1.5 P) s+(P-3), \\
a \leq \frac{1+(P-3) s^{2}+(2-P) s^{3}}{s^{4}-2 s^{3}+s^{2}},
\end{array}\right.
$$

From Eq. (14), it is established that the other coefficients $b, c, d, e$ can be established by a given $P, a$, so that the distribution of $g(s)$ is solved when the values of $P, a$ are determined. Moreover, according to the range of $s$, inequalities in Eq. (14) can be further simplified as:

$$
\left\{\begin{array}{l}
a \geq \max \left(f_{1}(s)\right)(0 \leq s<0.5), a \leq \min \left(f_{1}(s)\right)(0.5<s \leq 1) \\
a \leq \min \left(f_{2}(s)\right)(0<s<1), \\
f_{1}(s)=\frac{(3-1.5 P) s+(P-3)}{(s-1)(2 s-1)} \\
f_{2}(s)=\frac{1+(P-3) s^{2}+(2-P) s^{3}}{s^{4}-2 s^{3}+s^{2}}
\end{array}\right.
$$

where $\max \left(f_{1}(s)\right), \min \left(f_{1}(s)\right)$ and $\min \left(f_{2}(s)\right)$ in Eq. (15) are controlled by $P$, which is shown in Figure 2 . The data in Figure 2(b) reveal that: as $P$ ranges from 0 to 2.882, there is only one area marked in Figure 2(b) satisfying the constraint inequalities in Eq. (15), while, no other areas can meet the constraints with the change of $P$.

Overall, to satisfy the physical distribution law, the distribution of the swirl velocity along the streamline is eventually deduced as:

$$
\left\{\begin{array}{l}
r V_{\theta}(s)=r_{1} V_{\theta 1}+g(s)\left(r_{2} V_{\theta 2}-r_{1} V_{\theta 1}\right)(0 \leq s \leq 1), \\
g(s)=a s^{5}+(-2 a+P-2) s^{4}+(a-P+3) s^{3},
\end{array}\right.
$$

where $r_{1} V_{\theta 1}$ and $r_{2} V_{\theta 2}$ are calculated by the established variables from the target, and $(P, a)$ can be selected from the sail-like constrained area in Figure 2(b) to determine the swirl velocity distribution.

\subsection{Application of the Constrained Area in the Design Theory}

Based on the previous discussion, three cross sections were applied here to configure the runner blade of the

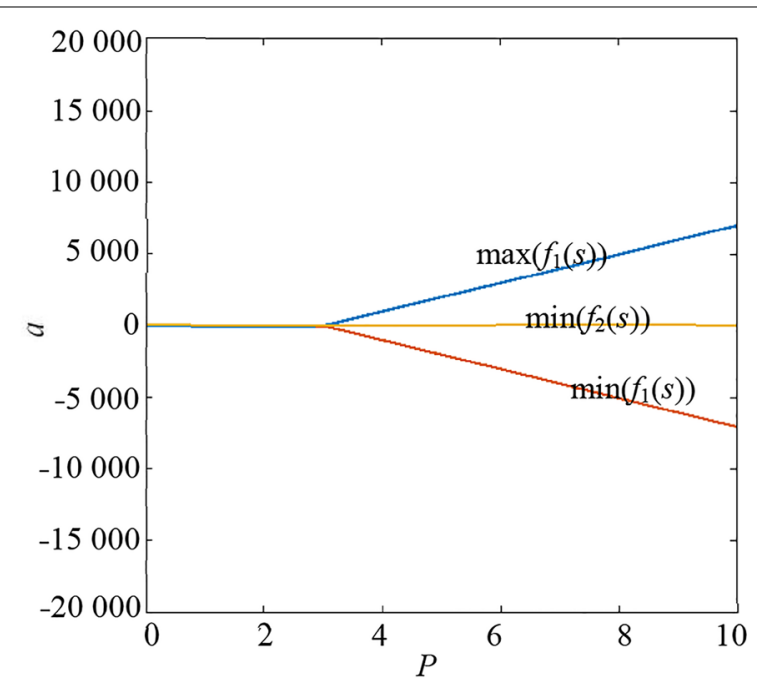

(a) $P \in[0,10](P \in[10,+\infty)$ has the same trend $)$

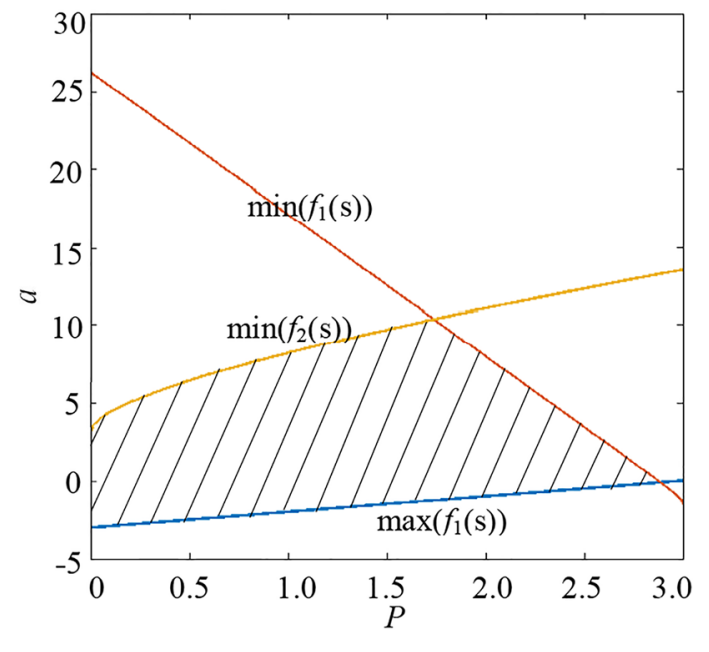

(b) $P \in[0,3]$

Figure 2 Derivation of the sail-like constrained area

mixed-flow pump, which is shown in Figure 3(a). The results indicate that each cross section is controlled by ten wrap angles. According to Eq. (2), the wrap angle of the $j$ th point for these cross sections can be calculated as:

$$
\left\{\begin{array}{l}
\theta_{h, j}=\sum_{i=1}^{j} \frac{\omega r_{h, i}^{2}-\left(r V_{\theta}(s)\right)_{h, i}}{V_{m, h, i} r_{h, i}^{2}} \\
\theta_{m, j}=\sum_{i=1}^{j} \frac{\omega r_{m, i}^{2}-\left(r V_{\theta}(s)\right)_{m, i}}{V_{m, m, i} r_{h, i}^{2}} \\
\theta_{s, j}=\sum_{i=1}^{j} \frac{\omega r_{s, i}^{2}-\left(r V_{\theta}(s)\right)_{s, i}}{V_{m, s, i} r_{s, i}^{2}}
\end{array}\right.
$$




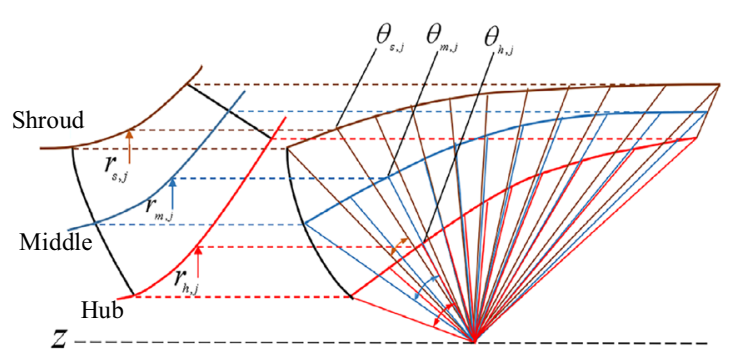

a Wrap angles at the cross sections [19]

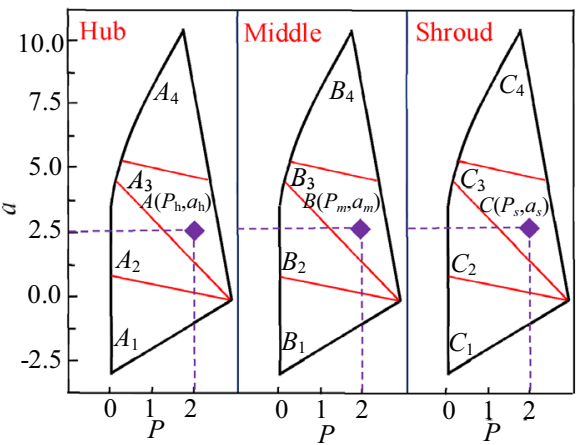

b Design variables from the sail-like constrained area

Figure 3 Wrap angles controlled by the design variables

where $\theta_{h, j}, \theta_{m, j}$ and $\theta_{s, j}$ are the wrap angles at the hub, middle and shroud side respectively.

The design variables to control the blade shape are given in Figure 3(b). The variables are selected from the sail-like constrained area, and they are then used to establish the swirl velocity with Eq. (16).

As previously mentioned, since the meridional shape, blade thickness, blade number, etc. from the target pump are settled, the preliminary averaged swirl velocity on the $S_{2 m}$ can be calculated first. Based on the calculated meridional velocity, the established swirl velocity and other previously settled variables, the wrap angles can be obtained. After some iterate calculations above, the blade shape would finally be determined when the wrap angles become stable. The flow-chart for the design method is shown in Figure 4.

\section{Multi-optimization System}

As shown in Figures 3(b) and 4, these design variables were randomly selected from the constrained area to generate the blade samples. Next, a multi-optimization strategy would be proposed to choose the right variables to optimize the runner blade. The previously reported design strategy $[11,24]$ is amended here to optimize the runner blade. The optimization strategy presented in Figure 5 consists of the modified inverse design method,

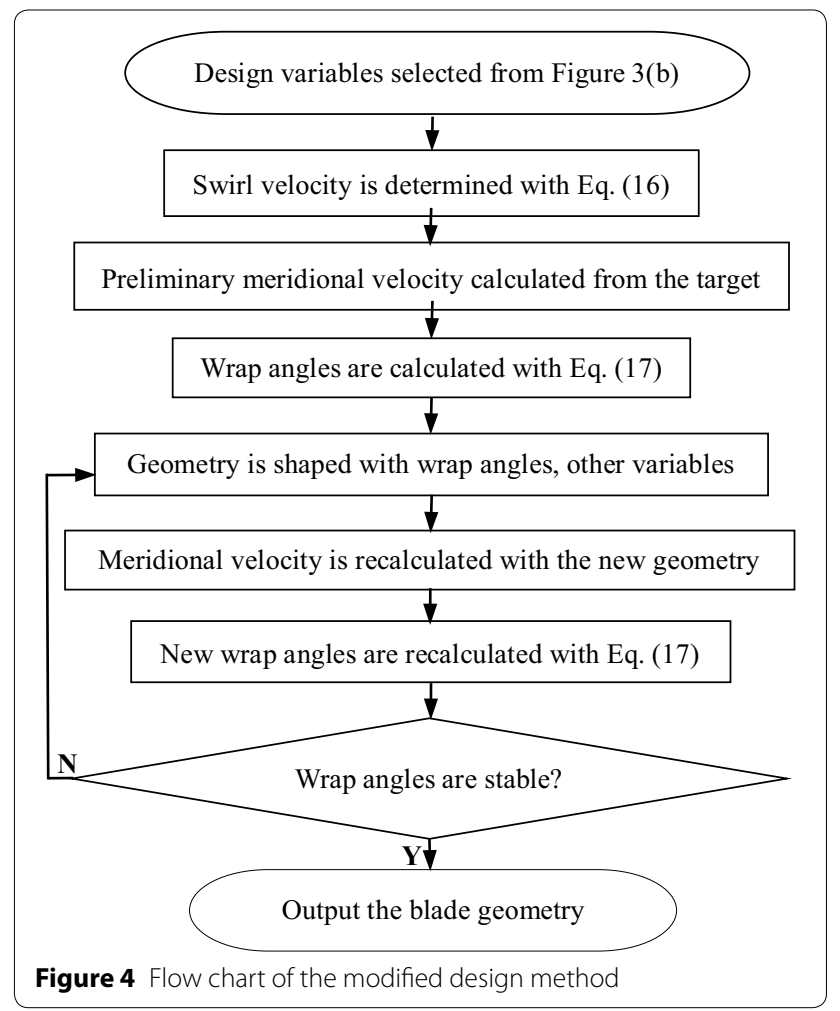

CFD analysis, RSM method and NSGA-II method. The details for the optimization process are as follows:

Step 1. Design targets that refer to the performances of the design impeller should catch up with the target pump at the set $0.8 Q_{d}, 1.0 Q_{d}$ and $1.2 Q_{d}$ mass flow conditions are set.

Step 2. The related design variables obtained from the target pump are input.

Step 3. A series of control design variables from the constrained area are selected to configure the blade shape by using the stratified sampling method and CFD analysis method.

Step 4. The mathematical relationships between the design variables and the objectives are established by using the RSM method.

Step 5. The right variables configuring the blade shape are chosen by applying the multi-objective algorithm NSGA-II.

\subsection{Computational Fluid Dynamics (CFD) Analysis 3.1.1 Mesh Generation}

After being configured by the inverse design method, the geometry was then imported into NUMECA/ Autogrid5. Taking into account the symmetry property 


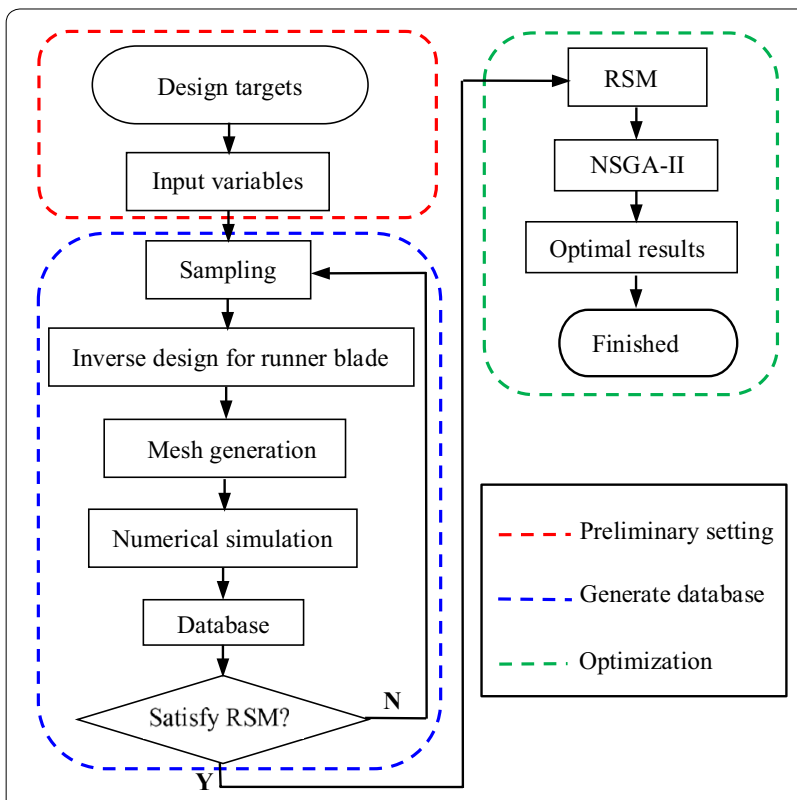

Figure 5 Flow chart of the multi-optimization system

of all blade passages in the pump, the flow characteristics of each blade passage can be considered to be the same. To reduce the grid number, it is necessary to select a single blade passage to simulate. The single blade passage is divided into several $\mathrm{H}$ blocks and I blocks with structured grids. To guarantee the quality, the meshed grids must have the right orthogonality $\left(>15^{\circ}\right)$, expansion factor $(<5)$ and aspect ratio $(<2000)$. Furthermore, to reach maximal $\mathrm{y}+$ around 10 in the computational domains, the minimum height of the cells is controlled to be $0.005 \mathrm{~mm}$ at the walls.

\subsubsection{Numerical Simulation}

The meshed grids are then imported into NUMECA/ Fine. To simulate the three-dimensional viscous compressible turbulent flow in the passage, the continuity equation, energy conservation equation and Reynolds Navier-Stokes equation processed by the turbulent model are combined. On account of the properties with good stability, small calculation and high accuracy, the Spalart-Allmaras turbulent model is chosen in the simulation process [21]. Moreover, with addition of a second and forth order artificial dissipation, the second-order central scheme is adopted. The time marching is performed in a four-stage Runge-Kutta scheme, coupled with local time stepping and implicit residual smoothing technologies for convergence acceleration.

\subsection{Optimization Process}

\subsubsection{Sampling Method}

Due to its high response of non-linear fitting and good ability to fill space [22], the stratified sampling method is adopted here in the sampling process. By applying the stratified method, control design variables are randomly selected in the constrained area, so that it can systematically and integrally sample each subpopulation. With the combination of the stratified method and the inverse design method, the sampling procedure is described as follows:

Step 1. As shown in Section 2.3, the constrained area at each cross section is divided into four equally small areas based on the equal area size. According to the data shown in this figure, it can be identified from the bottom to the top as: the small areas at the hub side, which are denoted as $A_{1}, A_{2}, A_{3}, A_{4}$ one by one; the small areas at the middle, which are identified as $B_{1}, B_{2}, B_{3}, B_{4}$; and the areas at shroud side, which are designated as $C_{1}, C_{2}, C_{3}, C_{4}$.

Step 2. Continually, from the sail-like area, the design variables $A\left(P_{h}, a_{h}\right), \quad B\left(P_{m}, a_{m}\right)$, $C\left(P_{s}, a_{s}\right)$ are randomly sampled from the split areas $A_{i}(i=1,2,3,4), \quad B_{j}(j=1,2,3,4)$, and $C_{k}(k=1,2,3,4)$ respectively. Based on the rule of permutation and combination, $4^{3}$ samples were obtained at the end, and the sample numbers are listed in the first row of the table found in the Appendix.

Step 3. After getting the sampled results of the design variables above, geometries for the runner blade were then configured with the inverse design method described in Section 2. Then, applying the CFD analysis method described in Section 3.1, the partial simulated performances of these samples were obtained and are also listed in the Appendix.

\subsubsection{RSM Method}

Regarding the engineering design process, it is almost impossible to exactly describe the functions between the input variables and output targets. Consequently, an approximate method must be used, and the RSM method is just the suitable one [8]. Accordingly, it is applied here to estimate the optimization targets. In order to make a relatively accurate description of the highly nonlinear relationships, the high order 
polynomial model is adopted. The RSM model is given by the following equation [20]:

$$
\begin{array}{r}
\hat{y}=\partial_{0}+\sum_{i=1}^{N} \partial_{1, i} x_{i}+\sum_{i=1}^{N} \partial_{2, i} x_{i}^{2}+\sum_{i=1}^{N} \partial_{3, i} x_{i}^{3}+ \\
\sum_{i=1}^{N} \partial_{4, i} x_{i}^{4}+\sum_{i=1}^{N} \partial_{5, i} x_{i}^{5}+\sum_{i=1}^{N} \partial_{6, i} x_{i}^{6},
\end{array}
$$

where $\hat{y}$ is the output target, $\partial$ denotes the coefficient, $N$ is number of input variables and equals 6 , the input variables are: $x_{1}$ is $P_{h}, x_{2}$ is $a_{h}, x_{3}$ is $P_{m}, x_{4}$ is $a_{m}, x_{5}$ is $P_{s}, x_{6}$ is $a_{s}$.

Generally, the prediction accuracy is highly dependent on the sample scale in the design space. As for the RSM model here, the least number of sample points is $S_{\min }=(N+1)(N+2) / 2$. And $N$ is 6 in this study, therefore, $S_{\min }$ is 28 . Since the number of samples in the database is 64 and exceeds 28 , the sample scale satisfies the RSM prediction.

\subsubsection{NSGA-II Method}

In view of the various conflicting optimization objectives, an effective multi-objective optimization algorithm is useful, and NSGA-II is just one of the ideal algorithms to perform this job. With the NSGA rooted in the arbitrary sharing code, Det [9] came up with an effective modified algorithm NSGA-II, which combines the elitistpreserving approach and the crowding distance operator to maintain uniform and sustainable diversity. Previous studies relevant to the multi-optimization of the pumps have been carried out applying the NSGA-II method [9, 20]. The NSGA-II method is mainly divided into the following steps.

Step 1. Initialize the random parent population of size $n$, and predict the optimized targets with the RSM method.

Step 2. The parent individuals are classified by the non-dominated rank and crowding distance.

Step 3. Individuals with higher crowding distance and lower rank are preferred in the mating pool for generating the next generation.

Step 4. Simulated binary crossover and polynomial mutation are then applied with the crossover probability of 0.9 and mutation probability of $1 / N$ ( $N$ is the number of variables, which is 6 here).

Step 5. After crossover and mutation, the mating pool generates a new generation of size $n$.

Step 6. Along with the preliminary population, the whole population (size $2 n$ ) is reduced to size $n$ according to their rank and crowding distance.

Step 7. Return to Step 2, and repeat the process until the fixed generation is reached.

\subsection{Optimization Targets}

To guarantee the working range of the new runner blade, the pump efficiencies at $0.8 Q_{d}, 1.0 Q_{d}$ and $1.2 Q_{d}$ are set as the optimization targets:

$$
\eta_{i}=\frac{\left(p t_{-} o u t_{i}-p t_{-} i n_{i}\right) \times Q_{i}}{\tau_{i} \times \omega} \quad(i=0.8,1.0,1.2),
$$

where $p t \_o u t, p t$ in are the total pressure at outlet and inlet, respectively, $Q$ is the volume flow, $\omega$ is the angular velocity, and $\tau$ is the torque.

Moreover, in order to improve the output energy, the head at the design point $1.0 Q_{d}$ should be considered at the same time. The head can be calculated as follows:

$$
H_{1.0}=\frac{p t_{-} \text {out }-p t_{-} \text {in }}{\rho g} .
$$

\section{Optimization of the Runner Blade}

\subsection{Introduction to the Target Pump and Mesh Scheme 4.1.1 Target Pump}

Regarding the target pump, it is taken from the core component of the scale hydraulic model 1400-MW canned nuclear coolant pump (on a scale of 1:2.5). The specific geometrical variables for the runner blade of the target mixed-flow pump are listed in Table 1. Also, the target pump has a rotation speed of $1495 \mathrm{r} / \mathrm{min}$, and its design mass flow is $Q_{d}=384 \mathrm{~kg} / \mathrm{s}$. The data shown in Figure 6(a) reveals that the target runner blade has a bend leading edge. The boundary conditions of the target pump are shown in Figure 6(a). Moreover, previous research [23] has established that water can be used as the fluid medium in the evaluation of the performance of canned nuclear coolant pump. The hydraulic model here and the previously reported model [24] are from the same series with a relatively high performance, so that other details for the target pump can be obtained from the published paper.

\subsubsection{Mesh Scheme and Its Validation}

The target mixed-flow pump is set as the simulation model, and to exclude the effects of the grids and prove the stability of the simulation method, its validation will be performed first. Under the operating conditions stated above, five kinds of grids ranging from 633548 to 1433940 are chosen to be simulated under the design condition. 
Table 1 Geometrical variables of the runner blade

\begin{tabular}{ll}
\hline Parameters & Values \\
\hline Inlet diameter $D_{1}(\mathrm{~mm})$ & 168 \\
Outlet diameter $D_{2}(\mathrm{~mm})$ & 320 \\
Wrap angle at hub $\theta_{h}\left({ }^{\circ}\right)$ & $0-130$ \\
Wrap angle at middle $\theta_{m}\left({ }^{\circ}\right)$ & $0-110$ \\
Wrap angle at shroud $\theta_{s}\left(^{\circ}\right)$ & $0-92$
\end{tabular}

In addition, these grids satisfy the quality requirements described in Section 3.1, and the solver items are set as described in the same Part. The computational convergence is set below $10^{-6}$. The calculations were conducted on a Dell Workstation with an Intel Core I5-6500 CPU.

The final simulation results of these grids are shown in Figure 6(b). According to the graph, when the number of grids exceeds 1261236, the simulation result would remain stable. Thus, this kind of grid would be the appropriate grid scheme in the multi-optimization system. According to the numerical simulation results, the pump's efficiency is $\eta_{\mathrm{d}}=90.4 \%$, and its head is $H_{d}$ $=22.1 \mathrm{~m}$. Taking the simulating performance of the target pump as the reference, the further optimization would be completed then.

\subsection{Results for the Optimization Process}

The results for each separate procedure, obtained through the application of the optimization process stated in Section 3, will be presented and discussed next. Using the sampling method in Section 3.2, Figure 7 shows the 64 generated samples in the database. Along with the performance of the samples, the wrap angle ranges at each cross section are also given in the table shown the Appendix. It can be inferred from these data that the modified design method could configure many blades with a wide range of blade shape, most importantly, these blades can also keep presenting good performances around the design point. Also, for these designed geometries with a relatively good performance, their wrap angles are increasing from the hub to the shroud gradually.

Additionally, through RSM analysis of the database presented in Figure 7, the mathematical relationships between the input design variables and the output performances were established. To show the reliability of RSM analysis, the R-square values are showed in Figure 8. As for RSM, the closer to 1 the R-squared value is,
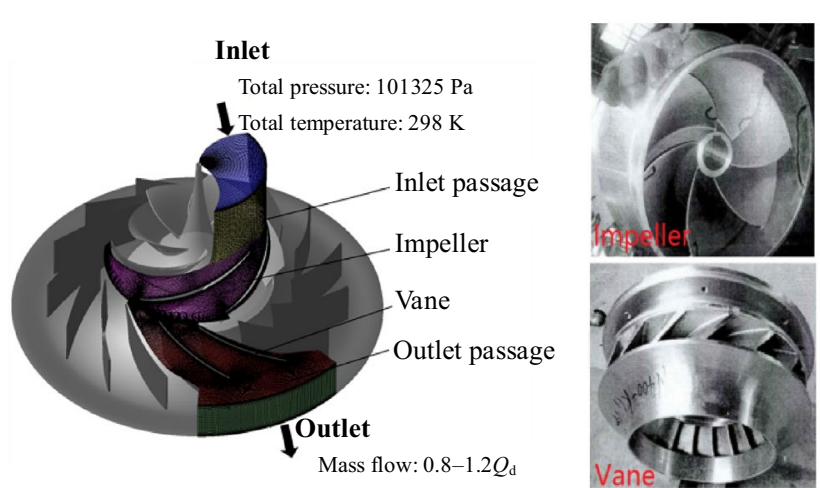

a Structure mesh distribution and the boundary conditions

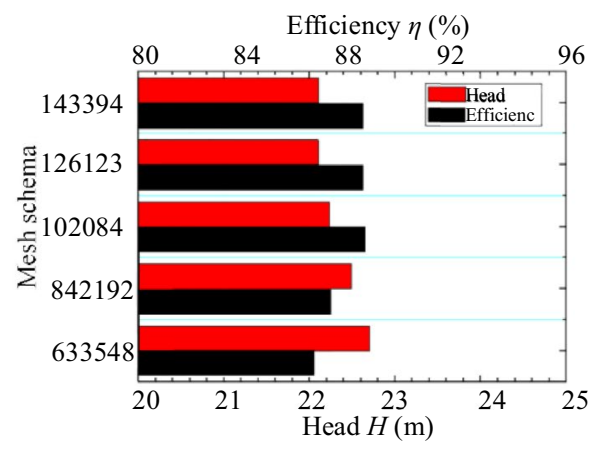

b Validation of various mesh schemes

Figure 6 Introduction of the target canned nuclear pump 

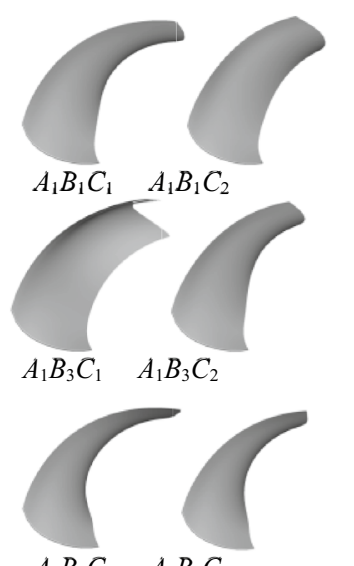

$A_{2} B_{1} C_{1} \quad A_{2} B_{1} C_{2}$

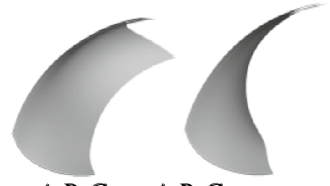

$A_{2} B_{3} C_{1} \quad A_{2} B_{3} C_{2}$
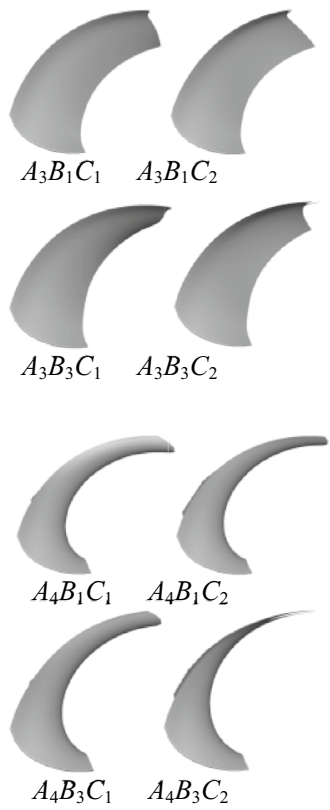

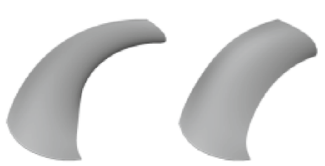

$$
A_{1} B_{1} C_{3} \quad A_{1} B_{1} C_{4}
$$

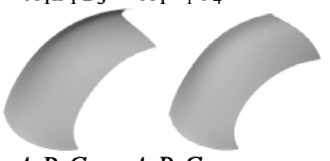

$A_{1} B_{3} C_{3} \quad A_{1} B_{3} C_{4}$

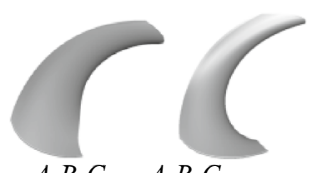

$A_{2} B_{1} C_{3} \quad A_{2} B_{1} C_{4}$

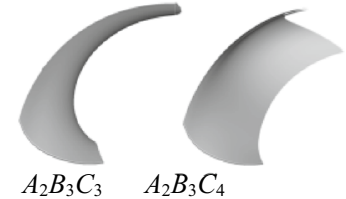

$A_{2} B_{3} C_{3} \quad A_{2} B_{3} C_{4}$

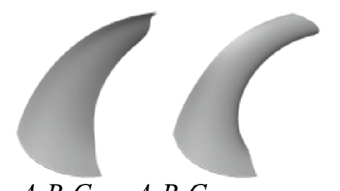

$A_{3} B_{1} C_{3} \quad A_{3} B_{1} C_{4}$
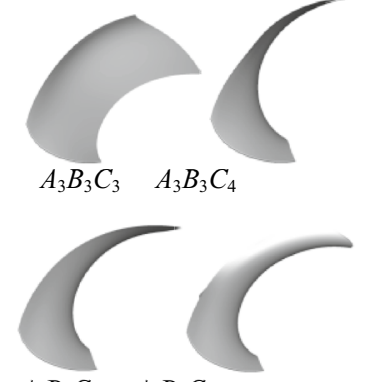

$A_{4} B_{1} C_{2} \quad A_{4} B_{1} C_{3}$

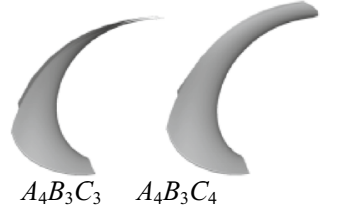

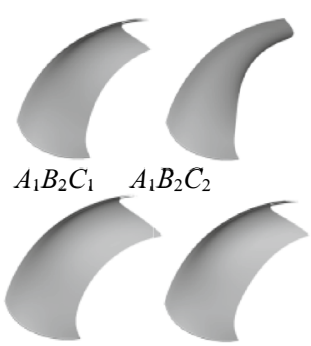

$A_{1} B_{4} C_{1} \quad A_{1} B_{4} C_{2}$
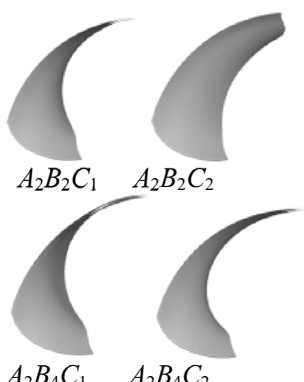

$A_{2} B_{4} C_{1} \quad A_{2} B_{4} C_{2}$

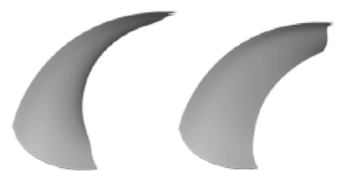

$A_{3} B_{2} C_{1} \quad A_{3} B_{2} C_{2}$

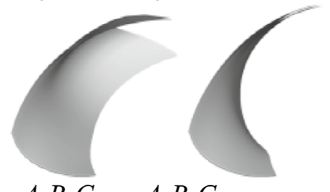

$A_{3} B_{4} C_{1} \quad A_{3} B_{4} C_{2}$

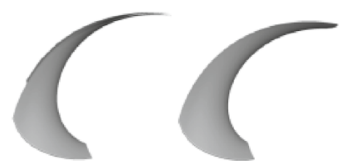

$A_{4} B_{2} C_{1} \quad A_{4} B_{2} C_{2}$

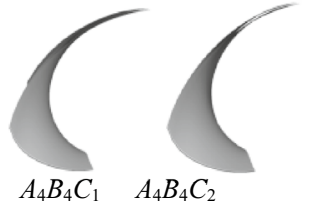

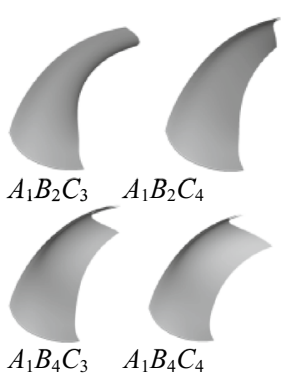

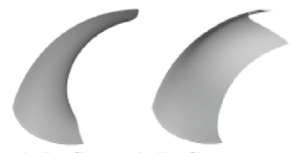

$A_{2} B_{2} C_{3} \quad A_{2} B_{2} C_{4}$
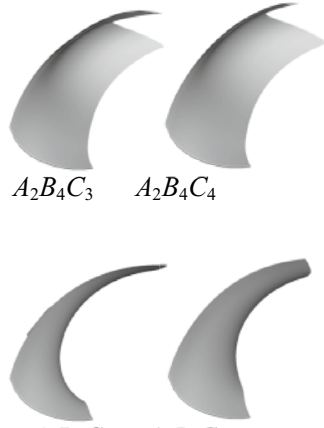

$A_{3} B_{2} C_{3} \quad A_{3} B_{2} C_{4}$

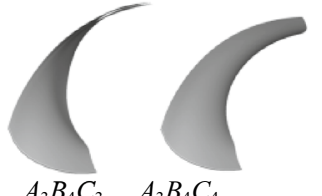

$A_{3} B_{4} C_{3} \quad A_{3} B_{4} C_{4}$

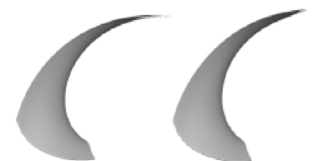

$A_{4} B_{2} C_{3} \quad A_{4} B_{2} C_{4}$

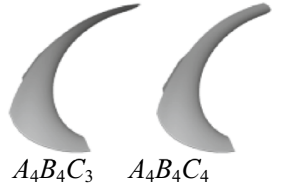

Figure 7 Designed runner blades in the database

the higher the accuracy of the RSM model would be. The $\mathrm{R}$-square values here are greater than 0.9 , which indicates that the RSM has a faithful prediction accuracy. After obtaining the RSM functions, they are then applied in the optimization system along with the NSGA-II algorithm.

The basic setting of the NSGA-II algorithm is shown in Table 2. Apart from this basic setting listed in the NSGA-II, the design variables are $A\left(P_{h}, a_{h}\right), B\left(P_{m}, a_{m}\right)$ and $C\left(P_{s}, a_{s}\right)$, which are selected from the sail-like constrained area of Section 2.3. Moreover, the efficiencies at $0.8 Q_{d}, 1.0 Q_{d}, 1.2 Q_{d}\left(\eta_{0.8}, \eta_{1.0}\right.$ and $\left.\eta_{1.2}\right)$ and head at design point $\left(H_{1.0}\right)$ are selected to be the predicted performances of the generated samples in the optimization process. To enlarge the number of effective output samples, the constraints for the predicted performances are also considered as follows: $0 \leq \eta_{0.8} \leq 1,0 \leq \eta_{1.0} \leq 1$, $0 \leq \eta_{1.2} \leq 1$, and $H_{d} \leq H_{1.0} \leq 1.15 H_{d}$. The optimization objectives are the efficiency of the target pump at the three mass flow points, as shown in Table 3 , their values are: $\eta_{t-0.8}=79.7 \%, \eta_{t-1.0}=90.4 \%, \eta_{t-1.2}=88.1 \%$. 


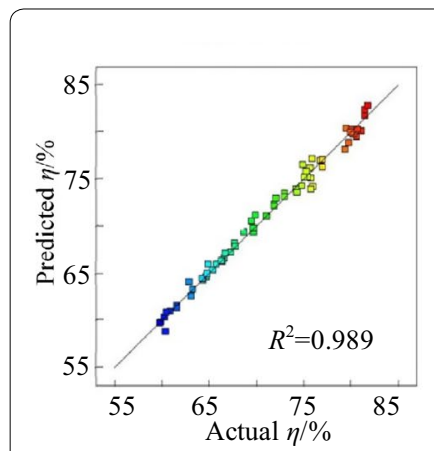

a Efficiency of $0.8 Q_{d}$ point

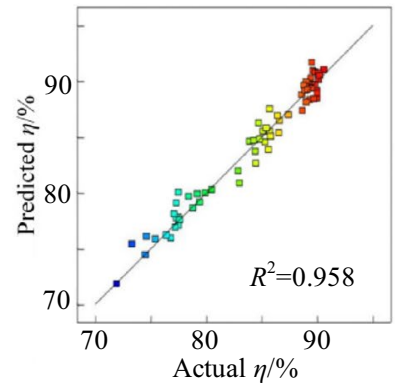

b Efficiency of $1.0 Q_{d}$

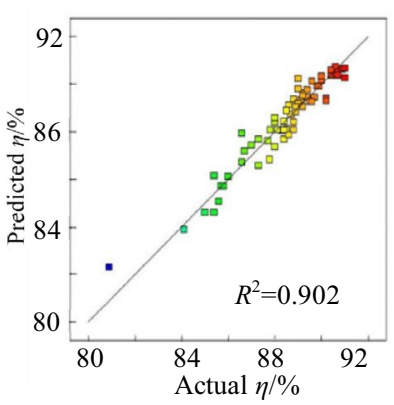

c Efficiency of $1.2 Q_{d}$

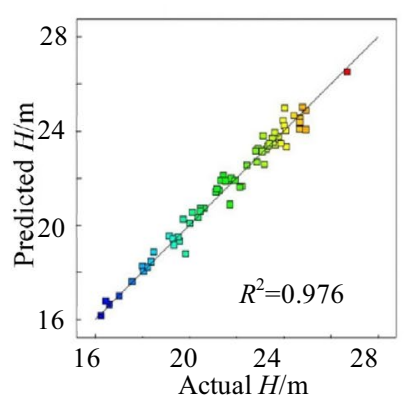

d Efficiency of $1.2 Q_{d}$

Figure 8 Analysis of the R-square error of RSM

Table 2 Basic setting of the NSGA-II

\begin{tabular}{ll}
\hline Parameters & Values \\
\hline Number of generations & 50 \\
Population size & 20 \\
Crossover probability & 0.9 \\
Crossover distribution index & 10 \\
Mutation distribution index & 20 \\
Initialization mode & Random \\
Max failed runs & 5 \\
\hline
\end{tabular}

The NSGA-II process was finally executed using the settings above, and a total of 1000 different samples were ultimately obtained. To choose the optimal runner blades among these samples, a new combined variable was used to measure the efficiencies of $0.8 Q_{d}, 1.0 Q_{d}$ and $1.2 Q_{d}$ and is defined as follows:

$$
\eta_{r e}=\frac{\eta_{0.8}}{\eta_{\mathrm{t}-0.8}}+\frac{\eta_{1.0}}{\eta_{\mathrm{t}-1.0}}+\frac{\eta_{1.2}}{\eta_{\mathrm{t}-1.2}} .
$$

Setting the defined $\eta_{r e}$ as the $Y$-axis, and the predicted design head $H_{1.0}$ as the $X$-axis, the results shown in Figure 9(a) were obtained and represent all samples obtained from NSGA-II. As for the samples, according to whether their predicted efficiencies exceed the efficiency of the target pump, they could be classified into effective samples $\left(\eta_{0.8}>\eta_{t-0.8}\right.$ and $\eta_{1.0}>\eta_{t-1.0}$ and $\left.\eta_{1.2}>\eta_{t-1.2}\right)$ and ineffective samples $\left(\eta_{0.8} \leq \eta_{t-0.8}\right.$ or $\eta_{1.0} \leq \eta_{t-1.0}$ or $\left.p^{+}\right)$. From the results in Figure 9(a), it can be ascertained that about $8.0 \%$ of the samples' efficiencies exceed those of the 1000 samples of the target impeller, and the optimal samples would be among them.

Considering the predicted errors and to guarantee the improvement of the head on the off-design operating conditions near the design point, the optimal samples are to be chosen from the effective samples of a relatively

Table 3 Performances for the effective samples with relative high head

\begin{tabular}{|c|c|c|c|c|c|c|c|c|c|c|c|c|}
\hline & $A\left(P_{h}, a_{h}\right)$ & $B\left(P_{m}, a_{m}\right)$ & $C\left(P_{s}, a_{s}\right)$ & $\theta_{h}\left(^{\circ}\right)$ & $\theta_{m}\left(^{\circ}\right)$ & $\theta_{s}\left({ }^{\circ}\right)$ & Items & $\eta_{0.8}(\%)$ & $\eta_{1.0}(\%)$ & $\eta_{1.2}(\%)$ & $H_{0.8}(\mathrm{~m})$ & Thrust $T(\mathrm{kN})$ \\
\hline Target & - & - & - & $0-130$ & $0-110$ & $0-92$ & CFD & 79.7 & 90.4 & 88.1 & 22.1 & 6.1 \\
\hline \multirow[t]{2}{*}{ S-1 (Opt1) } & $(2.03,0.57)$ & $(1.08,3.75)$ & $(1.71,5.73)$ & $0-142$ & 0-101 & $0-84$ & RSM & 80.9 & 91.3 & 89.9 & 25.3 & - \\
\hline & & & & & & & CFD & 81.5 & 91.6 & 88.9 & 23.9 & 5.9 \\
\hline \multirow{2}{*}{$\begin{array}{l}\text { S-2 } \\
\text { (Opt2) }\end{array}$} & $(1.96,1.05)$ & $(1.75,1.78)$ & $(1.13,0.36)$ & $0-132$ & $0-115$ & $0-91$ & RSM & 80.7 & 91.8 & 90.8 & 25.3 & - \\
\hline & & & & & & & CFD & 81.2 & 92.0 & 89.3 & 24.7 & 6.2 \\
\hline \multirow{2}{*}{$\begin{array}{l}\text { S-3 } \\
\text { (Opt3) }\end{array}$} & $(1.85,1.04)$ & $(1.75,1.65)$ & $(1.22,-0.51)$ & $0-129$ & $0-116$ & $0-93$ & RSM & 80.2 & 91.1 & 90.9 & 25.2 & - \\
\hline & & & & & & & CFD & 80.3 & 90.5 & 89.1 & 24.4 & 6.0 \\
\hline \multirow[t]{2}{*}{ S-4 } & $(1.66,-0.07)$ & $(1.67,1.78)$ & $(0.34,3.26)$ & $0-141$ & $0-113$ & $0-82$ & RSM & 80.2 & 90.9 & 90.6 & 25.1 & - \\
\hline & & & & & & & CFD & 78.9 & 91.1 & 89.4 & 24.2 & 6.2 \\
\hline \multirow[t]{2}{*}{ S-5 } & $(1.70,0.40)$ & $(1.63,0.64)$ & $(0.94,0.19)$ & $0-133$ & $0-118$ & $0-91$ & RSM & 80.1 & 91.9 & 89.6 & 25.0 & - \\
\hline & & & & & & & CFD & 80.3 & 89.9 & 88.5 & 23.9 & 6.2 \\
\hline
\end{tabular}

Italic values indicate the failure to meet the target performance

$A\left(P_{h}, a_{h}\right)$ Variables generating the hub angles; $B\left(P_{m}, a_{m}\right)$ Variables generating the middle angles; $C\left(P_{s}, a_{s}\right)$ Variables generating the shroud angles 


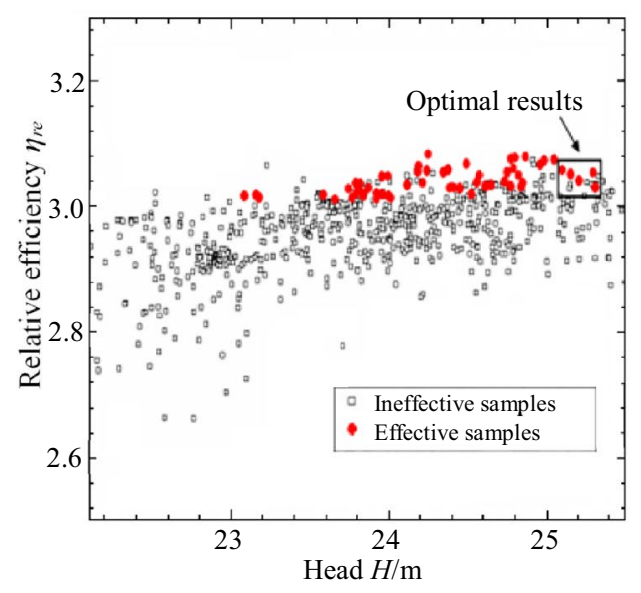

a Classification of the predicted samples

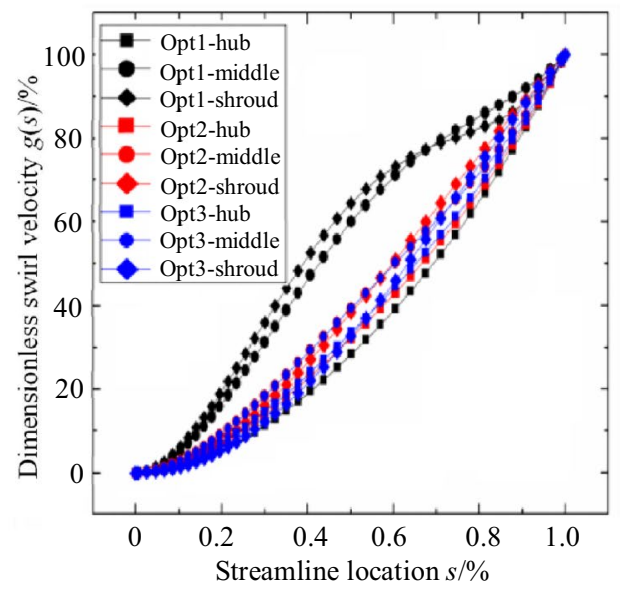

b The dimensionless swirl velocity of the optimal samples

Figure 9 Results obtained with the NSGA-II algorithm

high predicted design head $(25.0-25.5 \mathrm{~m})$. The predicted performances for these effective samples with relative high heads were verified by CFD analysis, and the results are listed in Table 3. Regarding the five samples with relatively high head shown in Figure 9(a), Table 3 shows their performances predicted by the RSM and simulated by CFD. From the results in Table 3, it is established that the efficiencies of three samples at the monitor mass flow points exceed those of the target mixed-flow pump. Therefore, these three samples are chosen as the optimal samples, which are named as Opt1, Opt2 and Opt3. Most importantly, in Table 3, the axial thrust of the optimal results are very close to the target, which implies that they would not lead to any safety problems in the new design. The dimensionless swirl velocity distribution of the optimal samples is shown in Figure 9(b). Their characteristics will be further discussed along with the target pump.

\subsection{Discussion of the Results}

\subsubsection{Experimental Validation for the Simulation}

Sample performances in the optimization process above are mainly got by CFD analysis, though Section 4.1 has already excluded out the effect of grids, its accuracy should be further discussed. Taking the target impeller as the test model, the experimental verification was conducted on the test rig in Shenyang Blower Works.

The experimental results and those obtained by CFD analysis are shown in Figure 10. As shown in Figure 10(b), the target pump is extracted from the full domain of CAP1400. The simulation performances of the full domain as well as the simplified structure are presented in Figure 10(c). These results reveal that when compared to the experimental results, the simulating results of the full domain are rather close to them, with results falling within an error of $1 \%$ for the efficiency, and an error of $5 \%$ for the head at the design point. As the efficiency at the design point is rather close to the experimental result, the simulation results for the whole domain can be approximately used to evaluate the losses by comparison of the simulation results of the simplified structure. Since the efficiency of the full domain is $85.2 \%$, while the efficiency of the simplified structure is $90.2 \%$, this clearly implies that about 5\% loss occurred there. Nevertheless, it can be observed from the results in Figure 10 that the experimental and the simulation results have the same ascend and descend trends. Moreover, the experimental efficiency curve and the simulation efficiency curve have the same design mass flow point. Thus, the CFD analysis is reliable and it can be effectively applied in the optimization process.

\subsubsection{Comparison of the Characteristics}

After the validation by CFD analysis, the simulation characteristics of the optimal samples and the target would be further discussed. The performance curves of the target pump and the optimal samples are presented in Figure 11(a). According to the results shown in Figure 11(a) and Table 3, as the operating condition ranges from $0.8 Q_{d}$ to $1.2 Q_{d}$ mass flow, the efficiencies of the optimal samples are improved when compared with those of the target pump, especially the maximum improvement in the design point, which can be as much as $1.6 \%$. Additionally, at the design mass flow point, the heads of these optimal samples are increased 


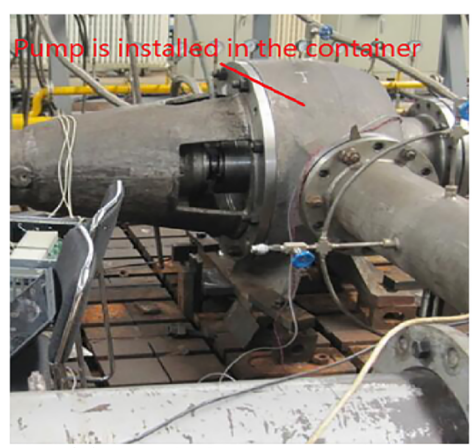

(a) Test rig for the target pump

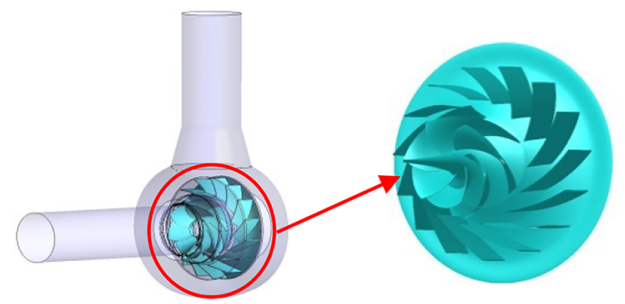

The full domain The simplified structure

(b) The target mixed-flow pump extracted from the full domain

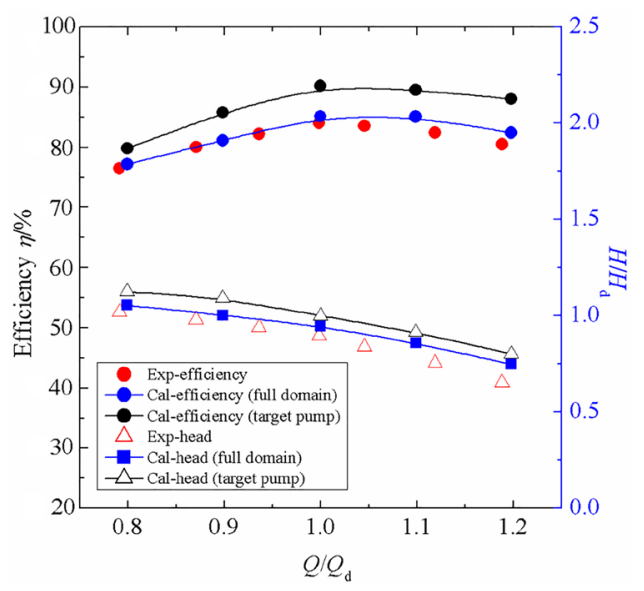

(c) Performance curves of the target pump

Figure 10 Experimental validation of the numerical simulations

by $2.0 \mathrm{~m}, 2.6 \mathrm{~m}$ and $2.1 \mathrm{~m}$, respectively, and the approximate head improvements can be as much as $10 \%$. To verify the performance improvement, the inner flow analysis for the optimal and the target would be conducted next.

The averaged meridional velocities on the $S_{2 \mathrm{~m}}$ surface are shown in Figure 11(b). These results indicate that that if the meridional shape, blade thickness, blade number, etc. from the target pump are settled, the average meridional velocities of the newly designed blades continue exhibiting a similar distribution compared with the target. Accordingly, meridional velocity of the target could be employed for the newly design blades as the initial before iterative design, which has already been discussed in Section 2.1.

The streamline distribution on the blade surface at the design point is shown in Figure 11(c). According to the results in this figure, there are four vortexes affecting the target pump's blade surface, however, the vortexes disappear in terms of the Opt1 blade, and the number of vortexes is decreased to three for the Opt 2 and Opt 3 blades. Most importantly, the vortex size is greatly decreased compared with the target. Since a vortex consists of a multitude of rotating flows, it really poses a threat to the stable inner flows and can also lead to unsteady forces on the runner blade $[25,26]$. Therefore, as for the optimal samples, the reduction of the vortexes would lead to a performance improvement of the inner flows relative to the target pump.

To verify the head improvement in Figure 11(a), the results in Figure 11(d) show the static pressure distribution of the impeller and vane at $50 \%$ blade height. According to the results in this figure, the static pressure of the optimal samples increases orderly from the inlet of the impeller to the outlet of the vane. Furthermore, regarding the optimal samples, the outlet static pressure is higher than the target pump (the color is much redder), which indicates that the optimal samples have a higher work capacity.

Apart from the discussion for the static pressure, Figure 11(e) shows the distribution of the quantitative total pressure along the streamline under the design condition. In this figure, at the outlet location, the total pressures of the optimal samples are larger than those of the target pump, which is consistent with the head improvement shown in Figure 11(a). Moreover, the pressure load of the optimal samples increased steadily along the streamline without any fluctuations, as shown in Figure 11(f), which means that the runner blades work on the flow medium continually without any distortions from the inlet to the outlet.

\section{Conclusions}

(1) For the modified inverse design method, a sail-like constrained area generating the design variables is derived after a series of mathematical simplifications. Using a small scale of variables from the constrained area, the blades are effectively configured.

(2) Setting the scale core component of a $1400-\mathrm{MW}$ canned nuclear coolant pump as the target, a multioptimization system is established on the modified inverse design method. The effectiveness of the optimization system is demonstrated after experimental verification and analysis of variance. 


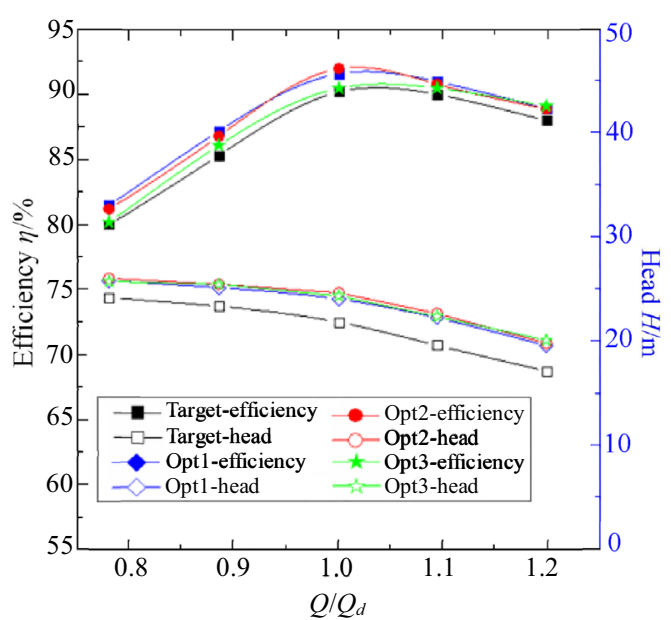

a Comparison of the performance curves
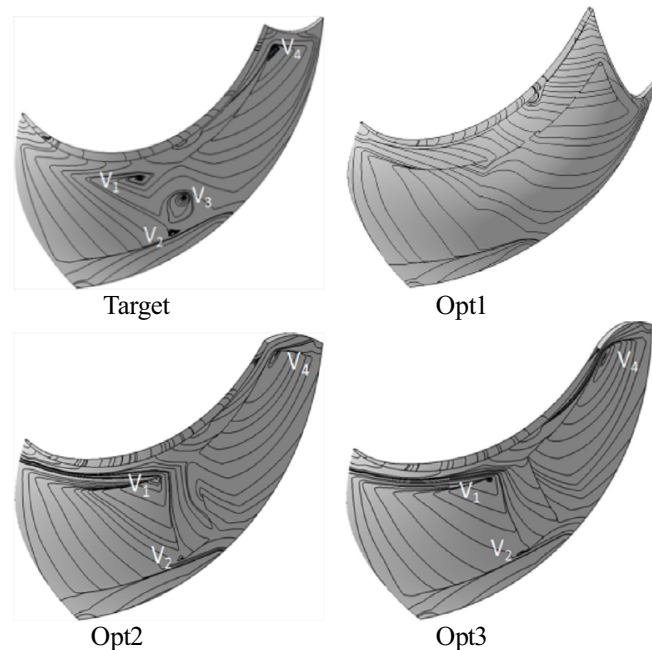

c Streamline located on the blade surface

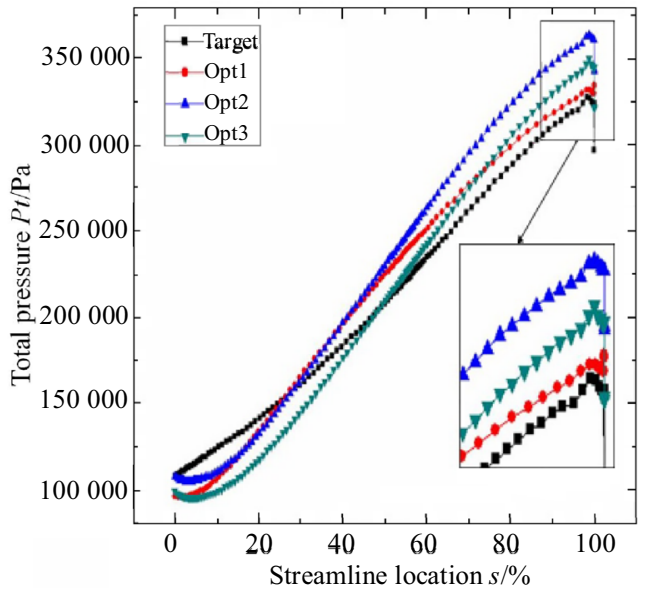

e Average total pressure distribution at $50 \%$ height
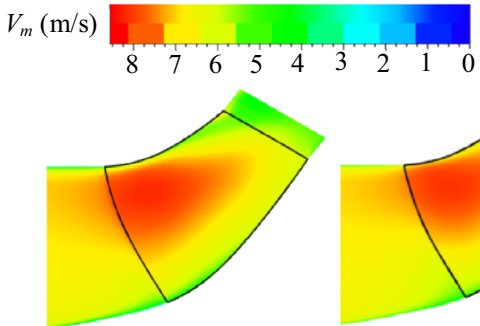

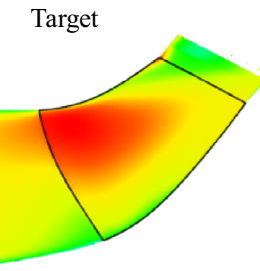

Opt2

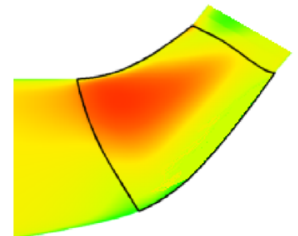

Opt1

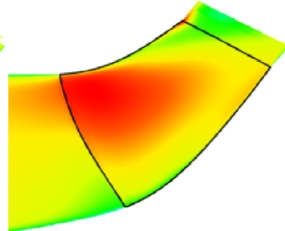

Opt3 b Averaged meridional velocities on the $S_{2 \mathrm{~m}}$ surface

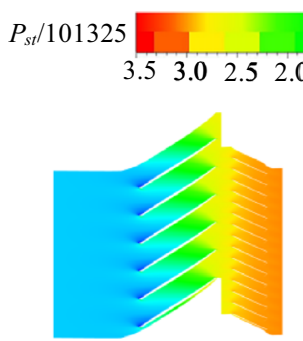

Target

Opt1

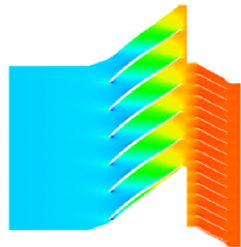

Opt2

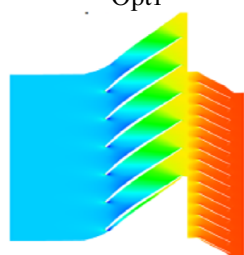

Opt3

d Static pressure distribution at 50\% blade height

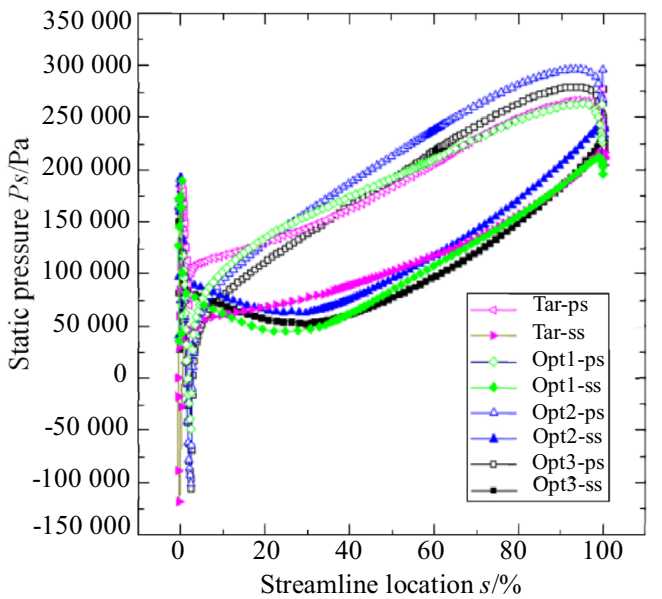

f Pressure load on the blade at $50 \%$ blade height

Figure 11 Comparison of the target and the optimal samples 
(3) The optimal samples are ultimately obtained after optimization. Through simulation and inner flow analysis, the performances are improved when compared to the target from $0.8 \mathrm{Qd}$ to $1.2 \mathrm{Qd}$ mass flow. At the design mass flow point, the maximum efficiency improvement is as much as $1.6 \%$, and the design head is improved by about $10 \%$.

\section{Authors' Contributions}

F-MZ and Y-ML were in charge of the whole trial; Y-ML wrote the manuscript; Y-ML, X-FW and WW assisted with sampling and laboratory analyses. All

authors read and approved the final manuscript.

\section{Author Details}

1 School of Energy and Power Engineering, Dalian University of Technology,

Dalian 116000, China. ${ }^{2}$ Key Laboratory of Ocean Energy Utilization and Energy

Conservation of Ministry of Education, Dalian University of Technology,

Dalian 116000, China. ${ }^{3}$ Collaborative Innovation Center of Major Machine

Manufacturing in Liaoning Province, Dalian 116000, China.

\section{Authors' Information}

Ye-Ming Lu, born in 1991, is currently a PhD candidate at School of Energy and Power Engineering, Dalian University of Technology, China. He received his master degree from Tianjin University, China, in 2016, and his bachelor degree from Northwestern Polytechnical University, China, in 2013. His research interests include pump design and inner flow analysis of the unshroud centrifugal impeller

Xiao-Fang Wang, born in 1961, is currently a professor at Dalian University of Technology, China. Her research interests include design and structural reliability analysis of turbomachinery.

Wei Wang, born in 1967, is currently an associate professor at Dalian University of Technology, China. Her research interests include the cavitation study and inner flow analysis of fluid machinery.

Fang-Ming Zhou, born in 1981, is currently a PhD candidate at School of Energy and Power Engineering, China. His research interests include the experimental investigation of the pump.

\section{Competing Interests}

The authors declare that they have no competing interests.

\section{Funding}

Supported by National Basic Research Program of China (973 Program, Grant No. 2015CB057301), Research and Innovation in Science and Technology Major Project of Liaoning Province, China (Grant No. 201410001), and Collaborative Innovation Center of Major Machine Manufacturing in Liaoning Province, China.

\section{Appendix}

\section{Nomenclature}

\begin{tabular}{ll}
\hline Symbols & Names \\
\hline$p^{+}$ & Static pressure on the pressure side \\
$p^{-}$ & Static pressure on the suction side \\
$B$ & Blade number \\
$V_{m}$ & Averaged meridional velocity \\
$\omega$ & Pump rotation velocity \\
$r V_{\theta}(s)$ & Swirl velocity distribution along \\
$r_{1} V_{\theta 1}$ & streamline \\
$r_{2} V_{\theta 2}$ & Swirl velocity at the blade inlet \\
$g(s)$ & Swirl velocity at the blade outlet \\
$a, b, c, d$ & Non-dimensional swirl velocity \\
$\beta_{1}$ & Coefficients determining $g(s)$ \\
$A\left(P_{h}, a_{h}\right)$ & Blade angle at the inlet \\
$\theta_{h, i}$ & Design point generating the hub \\
$B\left(P_{m}, a_{m}\right)$ & angles \\
$\theta_{m, i}$ & Wrap angles at the hub \\
$C\left(P_{s}, a_{s}\right)$ & Design point generating the middle \\
$\theta_{s, i}$ & angles \\
$\hat{y}$ & Wrap angles at the middle \\
$\eta$ & Design point generating the shroud \\
$H$ & angles \\
$p_{-} i n$ & Wrap angles at the shroud \\
$p_{-}$out & Output of the RSM \\
$\eta_{r e}$ & Efficiency of the pump \\
\hline & Toad of the pump \\
& Total pressure at the inlet of the \\
& Relative efficiency \\
&
\end{tabular}




\section{Part B Generated samples in the database}

\begin{tabular}{|c|c|c|c|c|c|c|c|c|c|c|c|}
\hline No. & $A\left(P_{h}, a_{h}\right)$ & $B\left(P_{m}, a_{m}\right)$ & $C\left(P_{s}, a_{s}\right)$ & $\begin{array}{l}\eta_{0.8} \\
(\%)\end{array}$ & $\begin{array}{l}\eta_{1.0} \\
(\%)\end{array}$ & $\begin{array}{l}\eta_{1.2} \\
(\%)\end{array}$ & $H_{1.0}(\mathrm{~m})$ & $\theta_{\mathrm{h}}\left({ }^{\circ}\right)$ & $\theta_{\mathrm{m}}\left(^{\circ}\right)$ & $\theta_{s}\left(^{\circ}\right)$ & $\begin{array}{l}\text { Thrust } T \\
\text { (kN) }\end{array}$ \\
\hline$A_{1} B_{1} C_{1}$ & $(0.263,-1.273)$ & $(1.323,-1.016)$ & $(1.569,-0.838)$ & 75.9 & 85.6 & 86.0 & 22.15 & $0-119$ & $0-122$ & $0-96$ & 5.2 \\
\hline$A_{1} B_{1} C_{2}$ & $(0.629,-1.287)$ & $(0.493,-0.185)$ & $(0.196,4.311)$ & 75.1 & 87.4 & 86.7 & 23.20 & $0-130$ & $0-112$ & $0-79$ & 5.8 \\
\hline$A_{1} B_{1} C_{3}$ & $(0.465,-0.727)$ & $(1.611,-0.774)$ & $(2.106,2.341)$ & 75.7 & 85.7 & 85.4 & 21.46 & $0-116$ & $0-124$ & $0-92$ & 4.8 \\
\hline$A_{1} B_{1} C_{4}$ & $(0.917,-1.176)$ & $(0.141,-1.344)$ & $(1.558,8.924)$ & 77.0 & 89.1 & 86.6 & 22.82 & $0-136$ & $0-113$ & $0-77$ & 4.7 \\
\hline$A_{1} B_{2} C_{1}$ & $(1.246,-0.313)$ & $(0.490,2.855)$ & $(0.087,-1.395)$ & 79.4 & 90.0 & 89.4 & 23.41 & $0-132$ & $0-99$ & $0-90$ & 5.6 \\
\hline$A_{1} B_{2} C_{2}$ & $(0.094,-1.319)$ & $(0.726,0.642)$ & $(0.275,1.911)$ & 74.8 & 88.6 & 88.7 & 24.70 & $0-115$ & $0-110$ & $0-84$ & 5.7 \\
\hline$A_{1} B_{2} C_{3}$ & $(0.493,-0.185)$ & $(1.386,2.241)$ & $(0.894,4.510)$ & 75.8 & 86.4 & 88.9 & 23.54 & $0 \sim 109$ & $0-110$ & $0-82$ & 6.6 \\
\hline$A_{1} B_{2} C_{4}$ & $(0.913,0.146)$ & $(0.413,3.121)$ & $(1.027,6.092)$ & 71.9 & 90.0 & 90.4 & 24.95 & $0 \sim 116$ & $0 \sim 97$ & $0-80$ & 6.3 \\
\hline$A_{1} B_{3} C_{1}$ & $(1.546,-0.158)$ & $(1.232,3.745)$ & $(0.165,-2.123)$ & 81.5 & 89.3 & 88.4 & 23.57 & $0-138$ & $0-102$ & $0-92$ & 6.2 \\
\hline$A_{1} B_{3} C_{2}$ & $(0.087,-1.395)$ & $(1.451,2.416)$ & $(0.110,3.043)$ & 76.8 & 88.9 & 88.9 & 24.81 & $0-116$ & $0-109$ & $0-81$ & 6.3 \\
\hline$A_{1} B_{3} C_{3}$ & $(0.528,-2.284)$ & $(1.679,3.709)$ & $(2.027,3.862)$ & 81.5 & 89.8 & 88.0 & 23.26 & $0-142$ & $0-106$ & $0-89$ & 6.1 \\
\hline$A_{1} B_{3} C_{4}$ & $(1.246,-1.374)$ & $(2.131,2.278)$ & $(1.833,8.256)$ & 80.0 & 88.8 & 85.6 & 21.19 & $0-149$ & $0-117$ & $0-83$ & 5.0 \\
\hline$A_{1} B_{4} C_{1}$ & $(1.483,-0.565)$ & $(1.994,5.056)$ & $(0.025,-0.823)$ & 81.8 & 90.1 & 88,6 & 22.45 & $0-143$ & $0-104$ & $0-88$ & 5.9 \\
\hline$A_{1} B_{4} C_{2}$ & $(0.412,-2.234)$ & $(2.110,6.355)$ & $(1.255,1.251)$ & 81.1 & 89.0 & 88.8 & 21.78 & $0-138$ & $0-100$ & $0-90$ & 5.1 \\
\hline$A_{1} B_{4} C_{3}$ & $(1.023,-0.032)$ & $(0.979,6.199)$ & $(1.187,4.725)$ & 73.0 & 89.0 & 90.6 & 24.45 & $0-121$ & $0-90$ & $0-83$ & 6.3 \\
\hline$A_{1} B_{4} C_{4}$ & $(1.513,-0.031)$ & $(1.256,5.705)$ & $(0.755,6.258)$ & 75.3 & 90.0 & 89.2 & 24.13 & $0-136$ & $0-95$ & $0-78$ & 5.9 \\
\hline$A_{2} B_{1} C_{1}$ & $(1.417,1.897)$ & $(0.730,-0.249)$ & $(2.518,-0.186)$ & 76.0 & 85.1 & 87.3 & 21.29 & $0-103$ & $0-114$ & $0-99$ & 6.4 \\
\hline$A_{2} B_{1} C_{2}$ & $(1.142,1.193)$ & $(0.219,0.025)$ & $(0.611,1.105)$ & 69.9 & 84.7 & 88.0 & 23.14 & $0-106$ & $0-108$ & $0-87$ & 5.5 \\
\hline$A_{2} B_{1} C_{3}$ & $(0.346,2.865)$ & $(1.313,0.216)$ & $(2.164,2.999)$ & 65.4 & 77.3 & 85.7 & 19.16 & $0-60$ & $0-117$ & $0-91$ & 5.5 \\
\hline$A_{2} B_{1} C_{4}$ & $(1.559,1.036)$ & $(0.212,-0.148)$ & $(1.825,5.965)$ & 75.8 & 89.3 & 89.4 & 24.70 & $0-121$ & $0-109$ & $0-84$ & 6.5 \\
\hline$A_{2} B_{2} C_{1}$ & $(0.722,1.155)$ & $(0.020,3.146)$ & $(0.257,-0.056)$ & 67.7 & 79.2 & 89.0 & 21.34 & $0-95$ & $0-93$ & $0-88$ & 5.2 \\
\hline$A_{2} B_{2} C_{2}$ & $(1.188,2.233)$ & $(0.526,1.420)$ & $(1.676,1.026)$ & 68.7 & 84.8 & 89.1 & 21.55 & $0-91$ & $0-105$ & $0-93$ & 5.3 \\
\hline$A_{2} B_{2} C_{3}$ & $(1.386,2.241)$ & $(0.502,1.069)$ & $(2.373,4.559)$ & 69.5 & 84.3 & 89.2 & 21.95 & $0-97$ & $0-107$ & $0-89$ & 6.5 \\
\hline$A_{2} B_{2} C_{4}$ & $(0.912,0.812)$ & $(0.054,2.925)$ & $(1.553,6.846)$ & 69.7 & 85.8 & 91.0 & 24.95 & $0-105$ & $0-95$ & $0-81$ & 6.2 \\
\hline$A_{2} B_{3} C_{1}$ & $(2.005,0.712)$ & $(1.280,4.859)$ & $(0.278,0.229)$ & 80.3 & 89.3 & 90.2 & 23.66 & $0-139$ & $0-98$ & $0-87$ & 6.3 \\
\hline$A_{2} B_{3} C_{2}$ & $(0.413,0.824)$ & $(0.223,4.813)$ & $(0.512,2.613)$ & 66.7 & 77.1 & 89.9 & 20.63 & $0-91$ & $0-89$ & $0-84$ & 4.8 \\
\hline$A_{2} B_{3} C_{3}$ & $(0.571,2.741)$ & $(1.642,3.552)$ & $(1.851,1.952)$ & 66.3 & 79.4 & 88.4 & 19.54 & $0-66$ & $0-107$ & $0-92$ & 5.2 \\
\hline$A_{2} B_{3} C_{4}$ & $(1.938,0.449)$ & $(0.650,4.111)$ & $(1.348,5.543)$ & 80.6 & 89.4 & 90.4 & 24.06 & $0-140$ & $0-96$ & $0-82$ & 5.9 \\
\hline$A_{2} B_{4} C_{1}$ & $(0.657,1.452)$ & $(1.523,8.375)$ & $(0.039,0.713)$ & 67.3 & 77.5 & 88.2 & 20.48 & $0-88$ & $0-86$ & $0-86$ & 7.7 \\
\hline$A_{2} B_{4} C_{2}$ & $(0.668,1.919)$ & $(1.867,6.503)$ & $(1.907,1.789)$ & 66.4 & 85.0 & 90.9 & 21.74 & $0-81$ & $0-97$ & $0-93$ & 6.1 \\
\hline$A_{2} B_{4} C_{3}$ & $(1.959,0.937)$ & $(1.404,6.751)$ & $(2.164,2.999)$ & 75.4 & 89.7 & 90.6 & 23.35 & $0-133$ & $0-92$ & $0-91$ & 5.4 \\
\hline$A_{2} B_{4} C_{4}$ & $(1.929,0.766)$ & $(0.954,5.832)$ & $(1.612,6.011)$ & 74.9 & 89.3 & 90.0 & 24.03 & $0-136$ & $0-91$ & $0-83$ & 6.8 \\
\hline$A_{3} B_{1} C_{1}$ & $(2.319,1.192)$ & $(0.501,-1.344)$ & $(0.976,-0.504)$ & 80.1 & 90.6 & 89.7 & 26.71 & $0-140$ & $0-116$ & $0-93$ & 6.8 \\
\hline$A_{3} B_{1} C_{2}$ & $(2.635,1.962)$ & $(0.045,-0.048)$ & $(0.275,1.911)$ & 80.7 & 89.8 & 88.9 & 23.64 & $0-137$ & $0-107$ & $0-84$ & 6.2 \\
\hline$A_{3} B_{1} C_{3}$ & $(2.031,2.207)$ & $(0.015,0.752)$ & $(1.025,3.351)$ & 71.9 & 86.6 & 89.5 & 24.69 & $0-116$ & $0-103$ & $0-85$ & 5.8 \\
\hline$A_{3} B_{1} C_{4}$ & $(2.436,3.973)$ & $(0.173,-0.400)$ & $(1.890,8.957)$ & 72.1 & 84.4 & 88.8 & 22.23 & $0-100$ & $0-109$ & $0-78$ & 4.9 \\
\hline$A_{3} B_{2} C_{1}$ & $(2.083,2.952)$ & $(0.512,0.785)$ & $(1.012,-1.463)$ & 71.1 & 85.4 & 89.0 & 22.93 & $0-106$ & $0-108$ & $0-96$ & 5.7 \\
\hline$A_{3} B_{2} C_{2}$ & $(2.165,1.407)$ & $(1.577,1.351)$ & $(2.118,1.007)$ & 79.8 & 89.5 & 89.2 & 23.99 & $0-132$ & $0-115$ & $0-95$ & 5.9 \\
\hline$A_{3} B_{2} C_{3}$ & $(1.246,4.246)$ & $(0.525,3.079)$ & $(2.352,2.546)$ & 62.9 & 83.7 & 88.5 & 19.85 & $0-62$ & $0-98$ & $0-93$ & 6.3 \\
\hline$A_{3} B_{2} C_{4}$ & $(1.921,2.699)$ & $(1.316,2.382)$ & $(1.809,4.943)$ & 74.2 & 85.7 & 88.6 & 22.87 & $0-105$ & $0-108$ & $0-86$ & 5.8 \\
\hline$A_{3} B_{3} C_{1}$ & $(2.059,1.498)$ & $(2.123,1.958)$ & $(2.517,-0.200)$ & 79.5 & 89.6 & 88.8 & 23.80 & $0-128$ & $0-117$ & $0-99$ & 6.0 \\
\hline$A_{3} B_{3} C_{2}$ & $(2.089,1.400)$ & $(1.920,4.141)$ & $(1.884,0.880)$ & 80.1 & 89.5 & 89.6 & 23.89 & $0-130$ & $0-107$ & $0-94$ & 6.1 \\
\hline$A_{3} B_{3} C_{3}$ & $(2.727,0.904)$ & $(2.395,3.463)$ & $(0.109,4.294)$ & 73.0 & 90.1 & 85.8 & 24.10 & $0-156$ & $0-114$ & $0-79$ & 5.7 \\
\hline$A_{3} B_{3} C_{4}$ & $(1.091,3.212)$ & $(0.354,5.193)$ & $(1.553,6.846)$ & 63.1 & 78.4 & 89.8 & 19.76 & $0-73$ & $0-88$ & $0-80$ & 5.6 \\
\hline$A_{3} B_{4} C_{1}$ & $(2.661,1.896)$ & $(1.341,6.847)$ & $(0.825,-1.371)$ & 77.0 & 90.2 & 90.0 & 23.10 & $0-139$ & $0-90$ & $0-94$ & 7.3 \\
\hline$A_{3} B_{4} C_{2}$ & $(1.862,3.886)$ & $(1.244,8.056)$ & $(0.500,3.945)$ & 65.8 & 76.4 & 90.2 & 20.04 & $0-85$ & $0-84$ & $0-81$ & 5.6 \\
\hline$A_{3} B_{4} C_{3}$ & $(1.712,3.286)$ & $(0.918,7.156)$ & $(1.523,4.845)$ & 67.8 & 77.5 & 91.0 & 20.47 & $0-90$ & $0-85$ & $0-85$ & 5.8 \\
\hline
\end{tabular}




\begin{tabular}{|c|c|c|c|c|c|c|c|c|c|c|c|}
\hline No. & $A\left(P_{h}, a_{h}\right)$ & $B\left(P_{m}, a_{m}\right)$ & $C\left(P_{s}, a_{s}\right)$ & $\begin{array}{l}\eta_{0.8} \\
(\%)\end{array}$ & $\begin{array}{l}\eta_{1.0} \\
(\%)\end{array}$ & $\begin{array}{l}\eta_{1.2} \\
(\%)\end{array}$ & $H_{1.0}(\mathrm{~m})$ & $\theta_{h}\left(^{\circ}\right)$ & $\theta_{\mathrm{m}}\left(^{\circ}\right)$ & $\theta_{s}\left({ }^{\circ}\right)$ & $\begin{array}{l}\text { Thrust } T \\
\text { (kN) }\end{array}$ \\
\hline$A_{3} B_{4} C_{4}$ & $(2.297,3.414)$ & $(2.152,4.868)$ & $(2.166,4.488)$ & 74.3 & 85.3 & 89.0 & 21.79 & $0-105$ & $0-106$ & $0-88$ & 5.6 \\
\hline$A_{4} B_{1} C_{1}$ & $(1.921,5.603)$ & $(1.120,0.094)$ & $(1.246,-0.351)$ & 64.8 & 80.5 & 85.4 & 19.58 & $0-60$ & $0-116$ & $0-93$ & 5.5 \\
\hline$A_{4} B_{1} C_{2}$ & $(1.335,5.055)$ & $(0.111,0.194)$ & $(2.023,0.424)$ & 59.9 & 77.6 & 88.0 & 18.24 & $0-52$ & $0-106$ & $0-95$ & 6.4 \\
\hline$A_{4} B_{1} C_{3}$ & $(2.265,4.598)$ & $(0.110,-0.545)$ & $(2.715,0.455)$ & 69.7 & 83.9 & 88.5 & 21.13 & $0-86$ & $0-109$ & $0-99$ & 6.5 \\
\hline$A_{4} B_{1} C_{4}$ & $(1.866,5.587)$ & $(1.103,-0.923)$ & $(2.174,5.232)$ & 64.3 & 79.9 & 84.1 & 19.32 & $0-58$ & $0-120$ & $0-87$ & 5.4 \\
\hline$A_{4} B_{2} C_{1}$ & $(2.125,6.027)$ & $(0.116,3.945)$ & $(2.185,0.178)$ & 61.6 & 76.8 & 87.3 & 16.62 & $0-59$ & $0-91$ & $0-97$ & 7.2 \\
\hline$A_{4} B_{2} C_{2}$ & $(2.013,4.835)$ & $(1.152,2.063)$ & $(2.833,0.023)$ & 66.6 & 86.6 & 87.7 & 20.39 & $0-74$ & $0-108$ & $0-10$ & 6.4 \\
\hline$A_{4} B_{2} C_{3}$ & $(2.223,5.135)$ & $(0.201,2.534)$ & $(2.574,1.232)$ & 64.9 & 83.1 & 89.6 & 19.36 & $0-76$ & $0-98$ & $0-97$ & 6.7 \\
\hline$A_{4} B_{2} C_{4}$ & $(2.215,4.735)$ & $(0.010,2.843)$ & $(2.185,5.322)$ & 64.7 & 77.3 & 90.7 & 20.14 & $0-82$ & $0-95$ & $0-87$ & 7.6 \\
\hline$A_{4} B_{3} C_{1}$ & $(1.875,5.326)$ & $(1.521,3.577)$ & $(1.902,5.504)$ & 64.4 & 78.8 & 87.8 & 18.50 & $0-63$ & $0-105$ & $0-85$ & 8.1 \\
\hline$A_{4} B_{3} C_{2}$ & $(1.922,5.935)$ & $(0.242,4.858)$ & $(1.512,1.755)$ & 60.5 & 74.6 & 88.1 & 17.03 & $0-55$ & $0-88$ & $0-91$ & 7.8 \\
\hline$A_{4} B_{3} C_{3}$ & $(1.350,5.027)$ & $(0.192,4.758)$ & $(2.212,2.545)$ & 60.4 & 73.3 & 88.3 & 16.47 & $0-52$ & $0-87$ & $0-92$ & 7.6 \\
\hline$A_{4} B_{3} C_{4}$ & $(1.745,5.526)$ & $(1.521,3.577)$ & $(1.902,5.504)$ & 63.3 & 77.5 & 88.4 & 18.07 & $0-56$ & $0-96$ & $0-85$ & 7.2 \\
\hline$A_{4} B_{4} C_{1}$ & $(1.621,4.926)$ & $(1.205,8.772)$ & $(0.025,0.351)$ & 61.6 & 71.9 & 80.5 & 16.27 & $0-62$ & $0-81$ & $0-88$ & 7.9 \\
\hline$A_{4} B_{4} C_{2}$ & $(2.213,4.946)$ & $(1.612,9.526)$ & $(0.127,3.092)$ & 60.9 & 74.5 & 85.0 & 17.58 & $0-78$ & $0-82$ & $0-81$ & 7.8 \\
\hline$A_{4} B_{4} C_{3}$ & $(1.945,5.758)$ & $(1.215,6.578)$ & $(1.025,3.845)$ & 60.3 & 77.2 & 87.0 & 18.39 & $0-58$ & $0-90$ & $0-84$ & 8.2 \\
\hline$A_{4} B_{4} C_{4}$ & $(1.221,4.826)$ & $(1.524,6.926)$ & $(1.313,8.556)$ & 59.8 & 75.4 & 86.5 & 18.02 & $0-51$ & $0-92$ & $0-76$ & 7.0 \\
\hline
\end{tabular}

\section{Publisher's Note}

Springer Nature remains neutral with regard to jurisdictional claims in published maps and institutional affiliations.

Received: 16 March 2017 Accepted: 16 November 2018 Published online: 21 December 2018

\section{References}

[1] J H Kim, B M Cho, S Kim, et al. Design technique to improve the energy efficiency of a counter-rotating type pump-turbine. Renewable Energy, 2017, 101: 647-659.

[2] S Kim, KY Lee, J H Kim, et al. High performance hydraulic design techniques of mixed-flow pump impeller and diffuser. Journal of Mechanical Science \& Technology, 2015, 29(1): 227-240.

[3] L H Liu, B S Zhu, L Bai, et al. Parametric design of an ultrahigh-head pump-turbine runner based on multiobjective optimization. Energies, 2017, 10(8): 1169

[4] J S Anagnostopoulos. A fast numerical method for flow analysis and blade design in centrifugal pump impellers. Computers \& Fluids, 2009, 38(2): 284-289.

[5] J E Borges. A three dimensional inverse method in turbomachinery: Part I-theory. ASME 1989 International Gas Turbine and Aeroengine Congress and Exposition, Toronto, Canada, June 4-8, 1989: V001T01A055-V001T01A055.

[6] M Zangeneh. A compressible three-dimensional design method for radial and mixed flow turbomachinery blades. International Journal for Numerical Methods in Fluids, 2010, 13(5): 599-624.

[7] P Wang, M Zangeneh, B Richards, et al. Redesign of a compressor stage for a high-performance electric supercharger in a heavily downsized engine. ASME Turbo Expo 2017: Turbomachinery Technical Conference and Exposition, Charlotte, USA, June 26-30, 2017: V008T26A027.

[8] B S Zhu, X Wang, L Tan, et al. Optimization design of a reversible pumpturbine runner with high efficiency and stability. Renewable Energy, 2015, 81: $366-376$.

[9] R F Huang, XW Luo, J Bin, et al. Multi-objective optimization of a mixedflow pump impeller using modified NSGA-II algorithm. Science China Technological Sciences, 2015, 58(12): 2122-2130.

[10] X J Li, Z Y Pan, D Q Zhang, et al. Centrifugal pump performance drop due to leading edge cavitation. IOP Conference Series: Earth and Environmental Science, London, UK, June 7-13, 2012: 032058.
[11] TTuzson. Centrifugal pump design. 1st ed. New York: John Wiley \& Sons, Inc., 1986.

[12] G Y Peng, S L Cao, M Ishizuka, et al. Design optimization of axial flow hydraulic turbine runner: Part I —an improved Q3D inverse method. International Journal for Numerical Methods in Fluids, 2002, 39(6): 517-531.

[13] G Y Peng, S L Cao, M Ishizuka, et al. Design optimization of axial flow hydraulic turbine runner: Part II-multi-objective constrained optimization method. International Journal for Numerical Methods in Fluids, 2002, 39(6): 533-548

[14] H Bing, S L Cao, L Tan, et al. Parameterization of velocity moment distribution and its effects on performance of mixed-flow pump. Transactions of the Chinese Society of Agricultural Engineering, 2012, 28(13): 100-105. (in (hinese)

[15] V Pachidis, I Templalexis, P Pilidis. A dynamic convergence control algorithm for the solution of two-dimensional streamline curvature methods. ASME Turbo Expo 2009: Power for Land, Sea, and Air, Florida, USA, June 8-12, 2009: 287-296.

[16] M Casey, C Robinson. A new streamline curvature throughflow method for radial turbomachinery. Journal of Turbomachinery, 2010, 132(3): 031021.

[17] S L Cao, G Y Peng, Z Y Yu. Hydrodynamic design of rotodynamic pump impeller for multiphase pumping by combined approach of inverse design and CFD analysis. Journal of Fluids Engineering, 2005, 127(2): 330-338.

[18] N X Chen, Y L Wu. Centrifugal pumps. Beijing: China Machine Press, 2002. (in Chinese)

[19] Y M Lu, X F Wang, R Xie. Derivation of the mathematical approach to the radial pump's meridional channel design based on the controlment of the medial axis. Mathematical Problems in Engineering. 2017, 2017(2): $1-16$.

[20] J Pei, W J Wang, S Q Yuan. Multi-point optimization on meridional shape of a centrifugal pump impeller for performance improvement. Journal of Mechanical Science and Technology, 2016, 30(11): 4949-4960.

[21] Y M Lu, Z X Liu. Quantitative analysis of influence factors on leakage flow of tip clearance in centrifugal impellers. Journal of Propulsion Technology, 2017, 38(1): 76-84. (in Chinese)

[22] G W Imbens, T Lancaster. Efficient estimation and stratified sampling. Journal of Econometrics, 1996, 74(2): 289-318.

[23] F M Zhou, X F Wang. Effects of staggered blades on the hydraulic characteristics of a 1400-MW canned nuclear coolant pump. Advances in Mechanical Engineering, 2016, 8(8): 1687814016657944 
[24] H Gao, F Gao, X C Zhao, et al. Analysis of reactor coolant pump transient performance in primary coolant system during start-up period. Annals of Nuclear Energy, 2013, 54(54): 202-208.

[25] S Kang. Numerical investigation of a high speed centrifugal compressor impeller. ASME Turbo Expo 2005: Power for Land, Sea, and Air, Nevada, USA, June 6-9, 2005: 813-821.
[26] D R Giosio, A D Henderson, J M Walker, et al. Design and performance evaluation of a pump-as-turbine micro-hydro test facility with incorporated inlet flow control. Renewable Energy, 2015, 78: 1-6.

\section{Submit your manuscript to a SpringerOpen ${ }^{\circ}$ journal and benefit from:}

- Convenient online submission

- Rigorous peer review

- Open access: articles freely available online

- High visibility within the field

- Retaining the copyright to your article

Submit your next manuscript at $\boldsymbol{\nabla}$ springeropen.com 